OPEN ACCESS

Edited by:

Ralph Panstruga,

RWTH Aachen University, Germany

Reviewed by:

Andrea Polle,

Georg-August-Universität Göttingen,

Germany

Sylvain Cordelier,

Université de Reims

Champagne-Ardenne, France

${ }^{*}$ Correspondence:

Eric M. Kemen

kemen@mpipz.mpg.de

Specialty section:

This article was submitted to Plant Biotic Interactions, a section of the journal Frontiers in Plant Science

Received: 6 January 2016 Accepted: 25 May 2016 Published: 20 June 2016

Citation:

Ruhe J, Agler MT, Placzek A,

Kramer K, Finkemeier I and Kemen EM (2016) Obligate Biotroph Pathogens of the Genus Albugo Are Better Adapted to Active Host Defense Compared to Niche Competitors. Front. Plant Sci. 7:820 doi: 10.3389/fpls.2016.00820

\section{Obligate Biotroph Pathogens of the Genus Albugo Are Better Adapted to Active Host Defense Compared to Niche Competitors}

\author{
Jonas Ruhe', Matthew T. Agler', Aleksandra Placzek ${ }^{1}$, Katharina Kramer ${ }^{1}$, \\ Iris Finkemeier ${ }^{1,2}$ and Eric M. Kemen ${ }^{1 *}$ \\ ${ }^{1}$ Max Planck Institute for Plant Breeding Research, Cologne, Germany, ${ }^{2}$ Institute of Plant Biology and Biotechnology, \\ University of Muenster, Münster, Germany
}

Recent research suggested that plants behave differently under combined versus single abiotic and biotic stress conditions in controlled environments. While this work has provided a glimpse into how plants might behave under complex natural conditions, it also highlights the need for field experiments using established model systems. In nature, diverse microbes colonize the phyllosphere of Arabidopsis thaliana, including the obligate biotroph oomycete genus Albugo, causal agent of the common disease white rust. Biotrophic, as well as hemibiotrophic plant pathogens are characterized by efficient suppression of host defense responses. Lab experiments have even shown that Albugo sp. can suppress non-host resistance, thereby enabling otherwise avirulent pathogen growth. We asked how a pathogen that is vitally dependent on a living host can compete in nature for limited niche space while paradoxically enabling colonization of its host plant for competitors? To address this question, we used a proteomics approach to identify differences and similarities between lab and field samples of Albugo sp.-infected and -uninfected $A$. thaliana plants. We could identify highly similar apoplastic proteomic profiles in both infected and uninfected plants. In wild plants, however, a broad range of defense-related proteins were detected in the apoplast regardless of infection status, while no or low levels of defense-related proteins were detected in lab samples. These results indicate that Albugo sp. do not strongly affect immune responses and leave distinct branches of the immune signaling network intact. To validate our findings and to get mechanistic insights, we tested a panel of $A$. thaliana mutant plants with induced or compromised immunity for susceptibility to different biotrophic pathogens. Our findings suggest that the biotroph pathogen Albugo selectively interferes with host defense under different environmental and competitive pressures to maintain its ecological niche dominance. Adaptation to host immune responses while maintaining a partially active host immunity seems advantageous against competitors. We suggest a model for future research that considers not only host-microbe but in addition microbe-microbe and microbe-host environment factors.

Keywords: biotrophy, Albugo, apoplast, proteomics, field studies, microbe-microbe interactions, systemic acquired resistance, immunity 


\section{INTRODUCTION}

In the wild, plants are exposed simultaneously to a variety of environmental stresses. Plant responses to biotic stress caused by microbes or abiotic stress like heat, starvation, or drought are largely distinct, but partly overlapping (Richards et al., 2012). In the laboratory, specific conditions are usually tested in isolation to study how plants react to stress on a molecular level. Ultimately, researchers would like to apply this knowledge to crop plants under field conditions and for this it is important to elucidate how laboratory-based knowledge transfers into nature.

Arabidopsis thaliana is the best-studied species of flowering plant (Koornneef and Meinke, 2010), but knowledge about how it behaves in the wild on a molecular level is still limited. This is because abiotic and biotic stimuli constantly fluctuate during wild plant growth, limiting replicability of field experiments. Under controlled conditions several studies examined how plants behave in response to single factors like salt and osmotic stress (Kreps et al., 2002), temperature change (Fowler and Thomashow, 2002; Kreps et al., 2002; Rizhsky et al., 2004) and drought (Rabello et al., 2008), starvation (Palenchar et al., 2004; Lian et al., 2006), or biotic stresses (Schenk et al., 2000; De Vos et al., 2005; Amrine et al., 2015; Lewis et al., 2015). Responses apparently interact, however, since gene expression analysis demonstrated that single stress responses cannot sufficiently predict changes after combined stresses (Mittler, 2006; Rasmussen et al., 2013). Therefore, to understand, for example, biotic stress responses, lab experiments with single stresses in isolation should be compared to the same stress under natural conditions.

The first studies on plant behavior under natural conditions showed that the transcriptome is mostly affected by circadian rhythm, environmental stimuli, and plant age (Nagano et al., 2012; Richards et al., 2012). Many gene clusters co-expressed under natural conditions are enriched in loci responsive to (a)biotic stimuli, which suggests that stress-responsive genes are deployed during the whole life cycle of $A$. thaliana (Richards et al., 2012). It is not clear yet how these transcription results translate into protein abundances in the field.

In the field, white blister rust, caused by the obligate biotroph oomycete genus Albugo, is one of the most widespread diseases of Brassicaceae (Ploch and Thines, 2011). Albugo sp. enter via stomata, form intercellular hyphae, penetrate the plant cell wall and invaginate the plant plasma membrane with haustoria in order to take up plant nutrients and release effector proteins into host cells (Kemen et al., 2005; Rafiqi et al., 2010; Spanu and Kämper, 2010; Kemen and Jones, 2012). To complete their whole lifecycle on the living host plant, obligate biotroph pathogens must be highly adapted to the host. Research on the effector complement of hemibiotroph and biotroph pathogens has already provided insights into how efficiently these pathogens interact with their respective host (De Wit et al., 2009; Caillaud et al., 2013; Asai et al., 2014; Irieda et al., 2014). For example, the biotrophic downy mildew pathogen Hyaloperonospora arabidopsidis ( $H p a)$, which has an overlapping host range with Albugo sp., and suppresses host responsiveness to salicylic acid (SA) in infected cells (Asai et al., 2014). It is known that hemi-biotrophic and necrotrophic pathogens trigger host secretion to the apoplast of defense-related proteins including pathogenesis-related (PR) proteins (Floerl et al., 2008, 2012; Ali et al., 2014; Kim et al., 2014). However, the influence of obligate biotroph pathogens on the $A$. thaliana apoplastic secretome is still unknown.

Host plant colonization by obligate biotrophs of the genus Albugo is associated with suppression of non-host resistance (NHR; Cooper et al., 2008). Specifically, Albugo sp. suppressed the "runaway cell death" phenotype, allowing a formerly avirulent downy mildew species to infect (Cooper et al., 2008). Assuming this phenomenon would extend to other non-host pathogens, Albugo sp. could thereby influence the microbial community composition of the host. We previously used network modeling of microbial community structures in the phyllosphere to show that the community composition is not only affected by abiotic factors and host genotype, but also by microbial hubs, including Albugo (Agler et al., 2016). Microbial hubs are taxa that are highly interconnected in a microbial community network and have significant effects on the community structure (Agler et al., 2016). It is still unclear whether hubs affect microbial colonization via direct microbe-microbe interactions or if they depend on host-mediated defense responses like suppression of NHR and effector-triggered immunity (ETI).

We investigated how Albugo, which is vitally dependent on a living host, can paradoxically compete in nature for a limited niche while in effect breaking host resistance to colonization. This was done by performing shotgun proteomics on leaf apoplastic fluid samples of Albugo sp.-infected and -uninfected A. thaliana. We examined plant samples from two different wild sites and a common garden experiment to compare the results with experiments performed under controlled laboratory conditions. Our results conclusively show that both wildgrown and lab-grown A. thaliana supports extensive Albugo sp. colonization, but the secretomes between wild and lab plants were significantly different. Regardless of Albugo sp. infection status, wild plants showed a broad spectrum of defense-related proteins at high abundances and lab-grown plants did not. We hypothesized that the activated immune system in wild plants leads to high hormone levels of, e.g., SA or abscisic acid (ABA). We used $A$. thaliana hormone mutants to mimic variable hormone levels in the field and found that Albugo laibachii strains are less affected in their infection rate than the natural competitor Hpa. Thus, our findings reveal how the biotroph pathogen Albugo only selectively interferes with host defense under different environmental conditions to maintain its ecological niche. Adaptation to host immune responses while maintaining partially active immunity seems advantageous against competitors.

\section{MATERIALS AND METHODS}

\section{Plant Growth Conditions}

Arabidopsis thaliana seeds were stratified on moist soil for 7 days at $4^{\circ} \mathrm{C}$ in darkness, before transfer into growth chambers with short day conditions ( $10 \mathrm{~h}$ light, $14 \mathrm{~h}$ darkness, $23 / 20^{\circ} \mathrm{C}$, constant 
$60 \%$ humidity). Plants were grown for 6 weeks prior to infection, except ABA mutants which were grown for 4 weeks. For apoplastic fluid proteomics experiments in the lab (see Extraction of Apoplastic Fluid from A. thaliana Leaves), A. thaliana Ws-0 was used. Since all mutants used for qPCR experiments (see DNA Extraction and Oomycete Growth Quantification via qPCR) were in Col-0 background, wild type Col-0 was used as a control for infections in these experiments.

\section{Arabidopsis thaliana Infections with Oomycete Pathogen Isolates}

Spore solutions were prepared by washing spores from Albugo candida Nc2 or A. laibachii Nc14 or MPI1 infected leaf material with tap water and held on ice for $1 \mathrm{~h}$. One milliliter spore solution was sprayed per plant $\left(16 \times 10^{4}\right.$ spores/ml $)$ on 6 -weekold A. thaliana using airbrush guns (Conrad Electronics $\mathrm{GmbH}$ ). Plants were kept overnight in a dark cold room to promote spore germination, then were further grown in cabinets (Sanyo Inc.) with a dark-light cycle of $10 \mathrm{~h}$ light at $22^{\circ} \mathrm{C}$ and $14 \mathrm{~h}$ darkness at $16^{\circ} \mathrm{C}$.

Infections with $\mathrm{Hpa}$ Noco2 were done similarly by washing infected leaves to make a conidiospore solution $\left(4 \times 10^{4}\right.$ spores $/ \mathrm{ml}$ ), then spraying on A. thaliana plants (Stuttmann et al., 2011).

\section{Sampling of Wild $A$. thaliana Plants}

Wild A. thaliana plants were sampled in Pulheim (Pul, $50^{\circ} 59^{\prime} 08.5^{\prime \prime} \mathrm{N} 6^{\circ} 49^{\prime} 35.4^{\prime \prime} \mathrm{E}$ ) and Geyen (Gey, $50^{\circ} 58^{\prime} 43.9^{\prime \prime} \mathrm{N}$ $6^{\circ} 47^{\prime} 35.8^{\prime \prime} \mathrm{E}$ ). Leaf material was pooled from several plants with white rust infection symptoms or asymptomatic plants at each site to reach appropriate amounts ( $\geq 2 \mathrm{~g}$ ) for apoplastic fluid extractions. While sampling asymptomatic plants, care was taken to use only leaves where the whole plant did not show any white rust sporulation. Sampling was done in spring and fall 2013 (only fall at Gey) and in spring 2014. A third (fourth) replicate was also taken from Gey (Pul) in spring 2015, but liquid chromatography tandem mass spectrometry (LC-MS/MS) analysis (see below) was performed with an updated workflow combining in-solution digestion and analysis on a Q Exactive Plus. This increased sensitivity, coverage and resolution; the absolute values are therefore not comparable with samples analyzed on the LTQ Velos after in-gel digestion.

Growth of plants in the common garden experiment at the Max-Planck-Institute, Cologne, Germany, was described in Agler et al. (2016). A. thaliana Ws-0 and Sf-2, which is an A. thaliana ecotype resistant to Albugo infections, were planted and sampling was performed exactly as described for the wild sites.

\section{Extraction of Apoplastic Fluid from A. thaliana Leaves}

For laboratory experiments, $\sim 7 \mathrm{~g}$ of leaves were harvested from uninfected mock $\left(\mathrm{H}_{2} \mathrm{O}\right.$ sprayed) or Albugo sp.infected $(10$ dpi) plants and immersed in $180 \mathrm{mM}$ 2-(N-morpholino)ethanesulfonic acid (MES) (Duchefa Biochemie; pH 5.5) infiltration buffer. Leaves sampled from natural sites or the common garden experiment (2-9 g) were washed twice with $\mathrm{ddH}_{2} \mathrm{O}$ to reduce dirt that was sticking to the leaves prior to immersion in infiltration buffer. A vacuum was applied with three cycles of 2:30 min followed by slow release over the course of $10 \mathrm{~min}$ to infiltrate the leaves homogenously. The leaves were blot-dried with tissue and placed in a $50 \mathrm{ml}$ falcon tube with holes in the bottom in a centrifugation bottle. Apoplastic fluid was collected via centrifugation (Thermo Scientific) at $4^{\circ} \mathrm{C}$ and $900 \times g$ for $10 \mathrm{~min}$ and was then sterile filtered $(0.22 \mu \mathrm{m}$ syringe filter, Spectrum Labs). Proteins were precipitated via chloroform/methanol precipitation (Wessel and Flügge, 1984). Pellets were dissolved in Laemmli sample buffer (Laemmli, 1970) for in-gel digestion and stored at $-20^{\circ} \mathrm{C}$ until LC-MS/MS analysis.

\section{LC-MS/MS Analysis}

For samples analyzed with the LTQ Velos machine (lab and wild samples replicates $1-3$ ), about $20 \mu \mathrm{g}$ of apoplast protein extract per sample was separated at $125 \mathrm{~V}$ on a linear $12 \%$ Tris-Glycine sodium dodecyl sulfate-polyacrylamide gel electrophoresis (SDSPAGE) for approximately $2 \mathrm{~h}$. Gels were stained with PageBlue protein staining solution (Fermentas). Each gel lane was dissected into 30-32 slices. Gel slices were destained, reduced, alkylated, and trypsinated by a Proteineer dp robot (Bruker Daltonics). Peptides were separated on a Thermo/Proxeon Easy nLC II in a two-column configuration (precolumn $3 \mathrm{~cm} \times 100 \mu \mathrm{m}, 5 \mu \mathrm{m}$ C18AQ medium, analytical column $10 \mathrm{~cm} \times 75 \mu \mathrm{m}, 3 \mu \mathrm{m}$ C18AQ) coupled to a LTQ-Velos ion trap (Thermo Scientific). Peptides were eluted over a segmented linear 130 min gradient running from 5 to $95 \%$ acetonitrile $(\mathrm{ACN}) / \mathrm{H}_{2} \mathrm{O}$ with $0.5 \%$ formic acid (FA) at a flow-rate of $300 \mathrm{nl} / \mathrm{min}$. Survey full-scan mass spectra were acquired in a mass range from 400 to $1600 \mathrm{~m} / z$. MS/MS spectra were acquired with collision-induced dissociation (CID) at $35 \mathrm{eV}$ on multiply charged precursor ions using a Top 10 method with active exclusion for $60 \mathrm{~s}$ in a window from $0.2 \mathrm{Da}$ below to $1.5 \mathrm{Da}$ above the precursor mass. The ion selection threshold was set to 500 counts for MS2, the activation Q was set to 0.25 and the activation time to $10 \mathrm{~ms}$. The resulting RAW files were converted to MGF format using Proteome discoverer 1.4.0288 (Thermo Scientific).

For samples analyzed on the Q Exactive Plus machine (lab samples, wild sample replicate 4 and common garden experiment samples), 5-20 $\mu \mathrm{g}$ apoplast protein extract per sample were digested in solution. The protein pellet was dissolved in $8 \mathrm{M}$ urea, $0.1 \mathrm{M}$ Tris- $\mathrm{HCl} \mathrm{pH}$ 8.0, $1 \mathrm{mM} \mathrm{CaCl}_{2}$. Cysteins were reduced by adding dithiothreitol (DTT) to a final concentration of $5 \mathrm{mM}$ and incubation for $30 \mathrm{~min}$. Subsequently, alkylation was performed by adding chloroacetamide to a final concentration of $14 \mathrm{mM}$ and incubation for $30 \mathrm{~min}$. The reaction was quenched by addition of DTT. Urea concentration was adjusted to $2 \mathrm{M}$ by dilution with $0.1 \mathrm{M}$ Tris- $\mathrm{HCl} \mathrm{pH} 8.0,1 \mathrm{mM} \mathrm{CaCl}_{2}$. Trypsin digestion (1:100 enzyme-to-protein ratio) was performed over night at $37^{\circ} \mathrm{C}$ and stopped by addition of $1 \%$ FA. Peptides were desalted with StageTips (Empore C18, $3 \mathrm{M}$ ) as described in Rappsilber et al. (2007). Dried peptides were redissolved in 2\% ACN, $0.1 \%$ trifluoroacetic acid for analysis and adjusted to a final concentration of $0.18 \mu \mathrm{g} / \mu \mathrm{l}$. Samples were analyzed using an EASY-nLC 1000 (Thermo Fisher) coupled to a Q Exactive Plus 
mass spectrometer (Thermo Fisher). Peptides were separated on $16 \mathrm{~cm}$ frit-less silica emitters (New Objective, $0.75 \mu \mathrm{m}$ inner diameter), packed in-house with reversed-phase ReproSil-Pur C18 AQ $3 \mu \mathrm{m}$ resin (Dr. Maisch). Peptides (1 $\mu \mathrm{g})$ were loaded on the column and eluted for 120 min using a segmented linear gradient of $0-95 \%$ solvent B (solvent A 5\% ACN, 0.5\% FA; solvent B 100\% ACN, 0.5\% FA) at a flow-rate of $250 \mathrm{nl} / \mathrm{min}$. Mass spectra were acquired in data-dependent acquisition mode with a Top15 method. MS spectra were acquired in the Orbitrap analyzer with a mass range of $300-1750 \mathrm{~m} / z$ at a resolution of 70,000 FWHM (full width at half maximum) and a target value of $3 \times 10^{6}$ ions. Precursors were selected with an isolation window of $1.3 \mathrm{~m} / \mathrm{z}$. High-energy collisional dissociation fragmentation was performed at a normalized collision energy of 25. MS/MS spectra were acquired with a target value of $10^{5}$ ions at a resolution of 17,500 FWHM and a fixed first mass of $\mathrm{m} / z$ 100. Peptides with a charge of +1 , greater than 6 , or with unassigned charge state were excluded from fragmentation for MS2, dynamic exclusion for $30 \mathrm{~s}$ prevented repeated selection of precursors.

\section{MS Data Processing}

MS/MS spectra were searched against the A. thaliana proteome (TAIR10) and against the A. laibachii Nc14/A. candida Nc2 (Kemen et al., 2011; McMullan et al., 2015) proteomes. A database containing 248 common contaminants and reverse "decoy" sequences were included. Trypsin specificity was required and a maximum of two missed cleavages allowed. Carbamidomethylation of cysteine residues was set as fixed modification and oxidation of methionine as variable modification. LTQ Velos data was searched using X! Tandem (version 2013.02.01.1; Craig and Beavis, 2004). A fragment monoisotopic mass error of $0.4 \mathrm{Da}$ was permitted with a parent monoisotopic mass error of $\pm 0.3 \mathrm{Da}$. Protein identifications were validated via the trans-proteomic-pipeline (TPP version 4.6.2; Deutsch et al., 2010), using default settings. For refinement and quantification of protein identifications APEX spectral counting (version 1.1.0) was used (Braisted et al., 2008). The APEX abundances of observed proteins were calculated using the results from PeptideProphet and ProteinProphet analyses (part of TPP) and a false discovery rate (FDR) of $1 \%$. For further analyses proteins were filtered for secreted proteins, based on TargetP 1.1 predictions (Emanuelsson et al., 2000) (Supplementary Material: Tables S3-S7).

Q Exactive data were processed using MaxQuant software (version 1.5.2.8 ${ }^{1}$; Cox and Mann, 2008) with label-free quantification (LFQ) and iBAQ enabled (Cox et al., 2014). Minimal peptide length was set to seven amino acids. Peptidespectrum-matches and proteins were retained if they were below a FDR of $1 \%$. Subsequent quantitative statistical analyses were performed in Perseus (version 1.5.2.6 ${ }^{1}$; Cox and Mann, 2012). LFQ intensities were $\log 2$ transformed. iBAQ values were used to filter for the 100 most abundant proteins, which were further analyzed based on their LFQ values (Supplementary Material: Table S8).

\footnotetext{
${ }^{1}$ http://www.maxquant.org
}

Due to different mass spectrometers and sample preparations a direct comparison based on protein abundances is not possible for samples analyzed with different setups.

\section{Statistical Analyses of Proteomics Samples}

To evaluate how the composition of proteomic samples varied between different treatments and sampling sites, we used redundancy analyses (RDA) in the R-package Vegan 2.2-1 (Oksanen et al., 2015). We first built an unconstrained model with the command RDA of all proteins in the sample with their respective APEX or LFQ abundance value. This model was then constrained by the factors location, infection status, or sample type (treatment). For visualization, we plotted the first constrained axes (if the factor was only two levels, only one constrained and one unconstrained axis are generated) and calculated the total variation of protein composition that was correlated to the constraining variables. To test if the constraining was significant $(p<0.05)$, we used the ANOVA function built into Vegan, which uses random permutations of factor classes followed by Tukey honestly significant difference (HSD) in R 3.0.2 (R Core Team, 2013). Ellipses of confidence intervals were plotted to help visualizing significant correlations. The built-in ordiellipse function was used to calculate the $95 \%$ confidence limits based on standard error.

RDA with all of the Q Exactive Plus-measured samples, constrained for the factor "treatments," indicated that five samples strongly diverged from their respective replicates (Supplementary Figure S1A). Due to technical reasons, these samples had abnormal MS/MS identification rates and showed low correlation to all other samples (Supplementary Figure S1B). For further analyses, these samples were excluded.

To determine if asymptomatic wild-grown $A$. thaliana plants were free of Albugo growth, the identified MS/MS spectra of these samples were searched against the A. laibachii Nc14 proteome and $A$. thaliana proteome and the ratio of Albugo proteins/A. thaliana proteins was compared with the ratios in Albugo-infected samples (Supplemenatry Tables S1 and S2).

\section{DNA Extraction and Oomycete Growth Quantification via qPCR}

Plants from the mutant infection assay were harvested at $10 \mathrm{dpi}$ (A. laibachii/A. candida infections or mock) or at 5 dpi (Hpa Noco2 infections or mock) and were immediately frozen in tubes in liquid nitrogen. Three adult plants (or five seedlings) were pooled and ground to powder using a liquid nitrogen-cooled mortar and pestle and DNA was extracted following a phenol/chloroform-extraction protocol (McKinney et al., 1995). In short, ground powder was taken, added to extraction buffer $(50 \mathrm{mM}$ Tris $\mathrm{pH} 8.0,200 \mathrm{mM} \mathrm{NaCl}$, $0.2 \mathrm{mM}$ ethylenediaminetetraacetic acid (EDTA), 0.5\% SDS, $0.1 \mathrm{mg} / \mathrm{ml}$ proteinase $\mathrm{K}$ (Sigma-Aldrich) and incubated at $37^{\circ} \mathrm{C}$ for $30 \mathrm{~min}$. One volume phenol was added, centrifuged and the top aqueous layer recovered and was mixed with 1 volume chloroform/isoamyl alcohol (24:1; Sigma-Aldrich). 
After centrifugation, the top aqueous layer was recovered and mixed with $3 \mathrm{M}$ sodium acetate and two volumes pure ethanol to precipitate the nucleic acids. DNA was pelleted by centrifugation and washed twice with $70 \%$ ethanol. It was resuspended in $50 \mu \mathrm{l}$ nuclease free water (NFW) and used for qPCR. DNA concentrations were determined via NanoDrop (Thermo Scientific) and diluted to $1 \mathrm{ng} / \mu \mathrm{l}$. One qPCR reaction contained $7.5 \mu \mathrm{l}$ SsoAdvanced universal SYBR Green supermix (Bio-Rad), $0.3 \mu \mathrm{l}$ of each primer $(10 \mathrm{mM})$, $1.9 \mu \mathrm{l} \mathrm{NFW}$ and $5 \mu \mathrm{l}$ DNA. Samples were measured in triplicates in a CFX Connect real-time PCR detection system (Bio-Rad) using the following program: (1) $95^{\circ} \mathrm{C}, 2 \mathrm{~min}$; (2) $\left(95^{\circ} \mathrm{C}, 20 \mathrm{~s}\right.$, then $56^{\circ} \mathrm{C}, 20 \mathrm{~s}$, then $\left.72^{\circ} \mathrm{C}, 30 \mathrm{~s}\right) \times 40,72^{\circ} \mathrm{C}$, 5 min followed by a temperature gradient from 65 to $95^{\circ} \mathrm{C}$. To quantify the amount of oomycete DNA per plant sample two standard genes were used (A. thaliana EF1- $\alpha$ : 5'-AAGGAG GCTGCTGAGATGAA-3', 5' -TGGTGGTCTCGAACTTCCAG3'; Oomycete internal transcribed spacer (ITS) 5.8s: $5^{\prime}$-ACTTT CAGCAGTGGATGTCTA-3', 5'-GATGACTCACTGAATTCTG CA- $\left.3^{\prime}\right)$. The amount of oomycete DNA was normalized to the respective plant DNA content and infected plant mutants were normalized to infected Col-0 wild type plants via calculating the $\Delta \Delta C q$.

\section{Amplicon Sequencing of Microbial Communities}

DNA extraction, amplicon library preparation, and sequencing were performed as previously described (Agler et al., 2016). In short, DNA was extracted with bead beating and SDS/lysozyme lysis with a phenol/chloroform cleanup. Amplification was performed for four amplicon regions (bacterial 16S rRNA gene regions V3/V4 and V5/V6/V7, Fungal ITS1 and ITS2) in two steps: The first step employed universal amplification primers and oligonucleotide clamps to block host amplification. The second step employed primers consisting of a concatenation of the Illumina adapter P5 (forward) or P7 (reverse), an index sequence (reverse only), a linker region, and the base primer for the region being amplified. Amplicon libraries were quantified fluorescently and products were combined in equimolar concentrations and were sequenced on an Illumina MiSeq lane using a mixture of custom sequencing primers complementary to the linker/primer region of the concatenated primers.

To process the reads, we used the custom pipeline described in Agler et al. (2016). For downstream analyses, operational taxonomic unit (OTU) tables were rarefied to an even depth of reads per sample and summarized to the genus level. A bar chart was plotted including all genera with $>5 \%$ abundance in any one sample (Supplementary Figures S2 and S3). Beta diversity plots were generated using principal coordinates analyses based on Bray-Curtis similarities between rarefied samples (Figure 5; Supplementary Figure S4). Alpha diversity (number of observed taxa) was calculated based on the average of 10 rarefactions of the data (Figure 5; Supplementary Figure S4). The raw sequencing data is available on Qiita².

\footnotetext{
${ }^{2}$ https://qiita.ucsd.edu/; study 10369
}

\section{Albugo Strain Determination with Microsatellite Markers}

Microsatellite markers were used to analyze genetic diversity between wild A. laibachii strains as in Agler et al. (2016). Three primer sets (AlSSR2[F/R], AlSSR6 [F/R], and AlSSR10 $[\mathrm{F} / \mathrm{R}]$ ) were employed which produce amplicons with which A. laibachii strains are easily distinguished. PCRs were performed as previously described (Agler et al., 2016) with equal amounts of extracted DNA from endophytic compartment samples as template. Products were visualized on a 3\% high-resolution agarose gel (Bio-Budget) with a 100 bp ladder. In some gels weak bands appeared in the background at lengths unexpected for the used markers. These were considered as non-target amplification and only bright bands of similar intensity were analyzed. If the length of amplified bands for all three markers were indistinguishable, we considered strains to be the same.

\section{RESULTS}

\section{The Secretome of Wild-Grown A. thaliana Differs Significantly from Lab-Grown Plants}

To get insights into the physiology of wild versus lab-grown plants we used a shotgun proteomics approach to elucidate plant secretomes on plants from two different wild sampling sites and from plants grown in a common garden experiment. We chose two sites nearby Cologne, Germany (Pul and Gey), due to their stable $A$. thaliana population structure and repeated observations of naturally occurring white rust symptoms caused by Albugo sp. These sampling sites are located within $2.5 \mathrm{~km}$ of one another and at similar elevations such that weathercaused environmental conditions are essentially similar. We sampled leaf material, pooled leaves from several plants at a site, during the early vegetative growth phase of $A$. thaliana in fall before its resting stage over winter and in spring, before it goes into its reproductive stage. All analyses are based on three biological replicates (two spring and one winter) from Pul, and two biological replicates (one spring and one winter) from Gey. We compared wild plants with or without visible white rust infection to lab-grown infected and uninfected mock plants. We focused our analyses on the plant apoplastic space, as it has been shown in previous work that upon biotic and abiotic stress perception there is a massive increase in secretion of defense- or stress-associated proteins into this compartment (Doehlemann and Hemetsberger, 2013; Delaunois et al., 2014). The apoplast is therefore a good analytical readout for plant responses.

Analyses revealed between 370 and 585 unique secreted Arabidopsis proteins per sample, with abundance values spanning four orders magnitude (Supplementary Material: Tables S3S7). We used constrained RDA of the 100 most abundant proteins across all uninfected samples to unravel the factors determining the variation of secretome compositions. Seventysix percent of the total protein variation (ANOVA based on 999 random class permutations for significance of the 


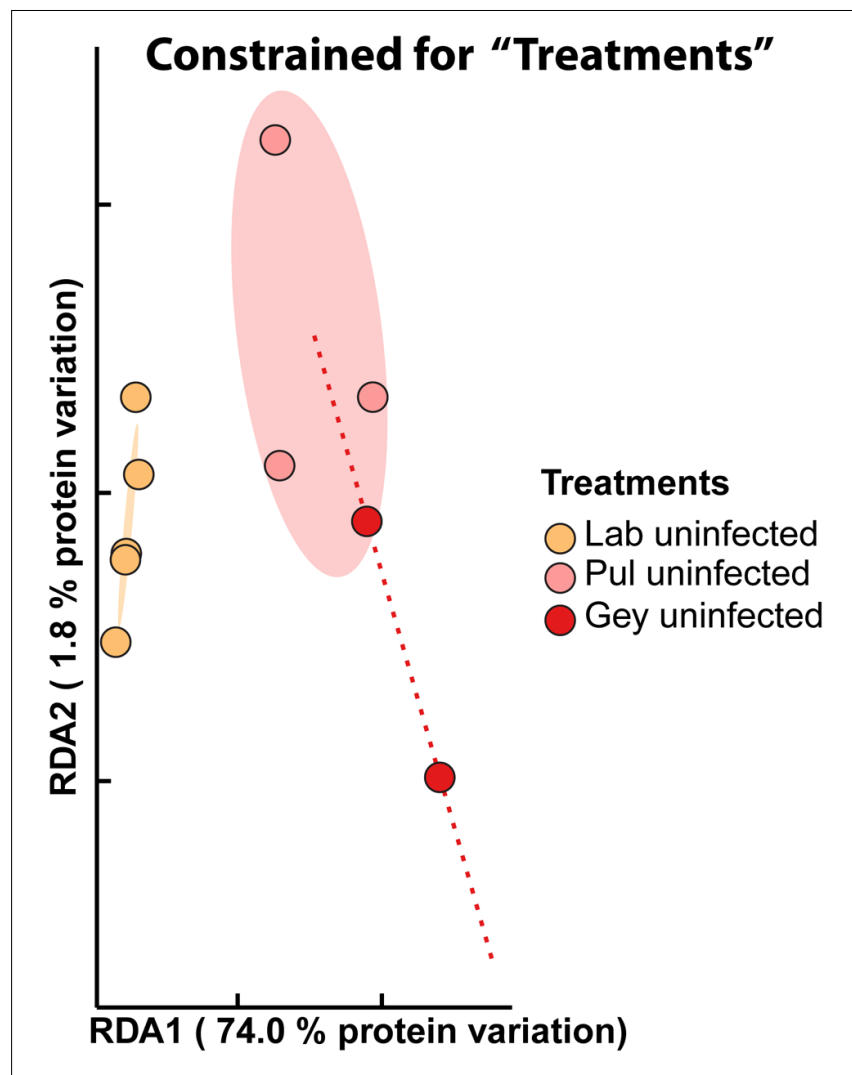

FIGURE 1 | The secretome of uninfected wild $A$. thaliana differs significantly from $A$. thaliana grown in controlled environment. The apoplastic fluid proteome (100 most abundant secreted proteins) of asymptomatic wild $A$. thaliana from Pul and Gey clusters significantly different to uninfected lab plants. Seventy-six percent of the variation in protein composition of these samples can be explained by constraining for these different "treatments" [lab uninfected (yellow, $n=5$ ), Pul uninfected (pink, $n=3$ ), Gey uninfected (red, $n=2)$ ] (ANOVA, $p$-value: 0.004). Confidence ellipses (lines) (0.95) based on the standard error show an overlap of Pul and Gey samples indicating their strong similarity.

treatment constraint, $p$-value: 0.004$)$ was constrained by the factor "treatments," distinguishing lab, Pul, and Gey samples (Figure 1). Here, uninfected lab samples cluster together and are significantly different from wild samples ( 0.95 confidence interval; Figure 1). Samples from different wild sites, however, show significant overlaps of their confidence intervals (Figure 1). This demonstrates that wild plants differ significantly from plants grown under controlled conditions even in an uninfected stage and that samples from two different wild sites are more similar to each other than to lab-grown plants.

To check if the phenotypically uninfected wild plants were free of Albugo sp. growth, we annotated the protein spectra against the $A$. laibachii $\mathrm{Nc14}$ genome and compared this with laboratory uninfected samples (Supplementary Table S1). Two of the five asymptomatic natural samples (Pul uninfected first and third replicate) exhibited augmented amounts of Albugo proteins (11.57\% respectively $14.37 \%$ ), while the other samples were comparable to background levels in non-inoculated control laboratory experiments (maximum 3.07\%). The higher levels in the two asymptomatic Arabidopsis samples could result from asymptomatic endophytic Albugo sp. growth (not visible during sampling) or contamination from Albugo spores attached to the leaves. However, they do not cluster closely together with corresponding samples showing white rust symptoms (Supplementary Figure S5C), suggesting that they do not behave like infected samples.

To demonstrate reproducibility of our experimental setup, we performed constrained ordination analyses with the factor "replicates" (three biological replicates) for all lab samples (uninfected and infected). Variation between replicates was not significantly more than random variation (ANOVA, $p$-value: 0.509), indicating the reproducibility of the results under controlled conditions (Supplementary Figure S6). On the other hand, "replicates" was a highly significant factor explaining most of the total variation (58\%) in wild samples (for both infected and uninfected samples; ANOVA, $p$-value: 0.004; Supplementary Figure S5C). Not surprisingly, the reproducibility of wild samples is low in comparison to the lab likely because the samples from different sampling dates and seasons (spring/fall) were exposed to different environmental stresses.

Considering the overall difference in secretome composition of uninfected wild and lab plants we had a closer look at the 100 most abundant proteins. Forty-two proteins had significantly different abundances between lab plants and wild Pul or Gey plants (Student's $t$-test, $p$-value $\leq 0.05$; Figure 2 ). For uninfected plants, the four overall most abundant proteins in the apoplastic fluid samples (annotated as: PR5 AT1G75040.1, PR1 AT2G14610.1, BG3 AT3G57240.1, PR2 AT3G57260.1) were significantly more abundant in the wild samples and are associated with responses to abiotic or biotic stimulus gene ontology (GO biological process, Berardini et al., 2004; Figure 2). In total, twelve proteins that had significantly higher abundance in the wild samples are associated with response to [a]biotic stimulus or to stress (GO biological process). This indicates that phenotypically healthy wild plants have an activated immune system which distinguishes them from plants grown in a controlled lab environment.

\section{The Oomycete Pathogen A. laibachii Does Not Suppress the Activated Immune System of Wild $A$. thaliana}

To determine to which extent the oomycete pathogen Albugo manipulates its host under controlled conditions and in the wild, we infected $A$. thaliana with two A. laibachii strains (isolates Nc14 and MPI1) and an A. candida strain (isolate Nc2). The strains $\mathrm{Nc} 14$ and Nc2 have been isolated from $A$. thaliana field plantings in Norwich, UK, while the MPI1 strain was the most frequently occurring Albugo sp. strain in our common garden experiments in Cologne, Germany (Kemen et al., 2011; McMullan et al., 2015; Agler et al., 2016). Microsatellite markers (van Treuren et al., 1997) demonstrated that in Pul and Gey A. thaliana plants were infected with various strains of $A$. laibachii whereas we did not $\operatorname{detect} A$. candida with specific primers in any wild or common garden experiment (Supplementary Figure S7 and Agler et al., 2016, for the common garden experiment). 


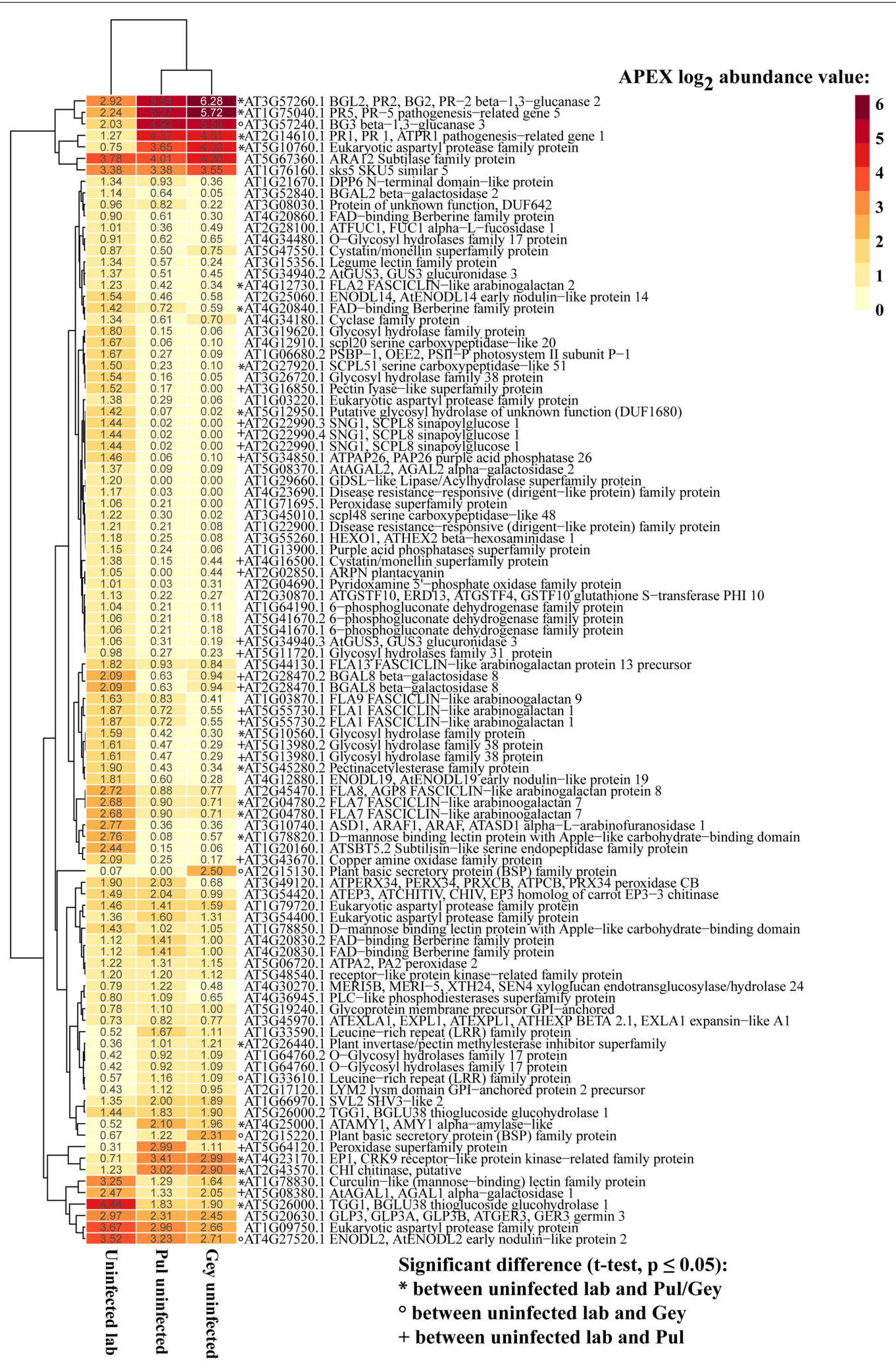

FIGURE 2 | Phenotypically uninfected wild $\boldsymbol{A}$. thaliana plants have high abundances of defense-related proteins. Heatmap of the 100 most abundant secreted $A$. thaliana proteins detected in uninfected lab and wild apoplastic fluid samples. The four most abundant proteins were significantly more abundant in wild plants and pathogenesis related. APEX abundance values were averaged across replicates and $\log _{2}$ transformed. Significant differences between samples are indicated by symbols. 
A

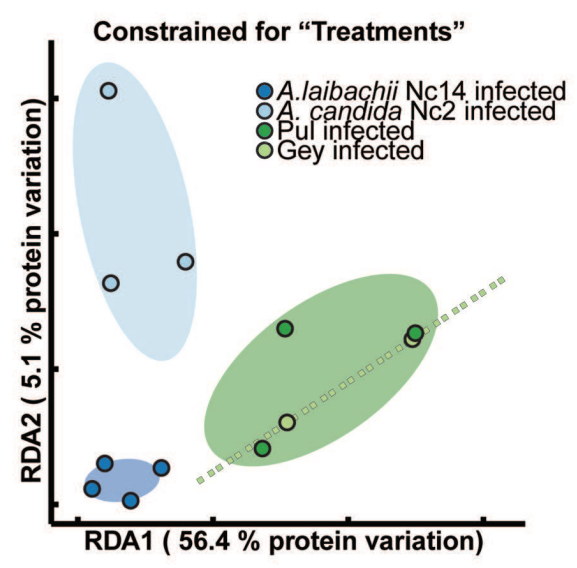

C

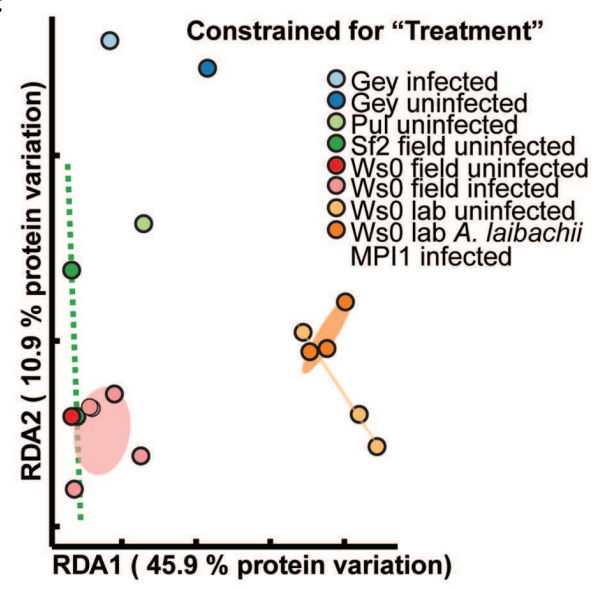

B

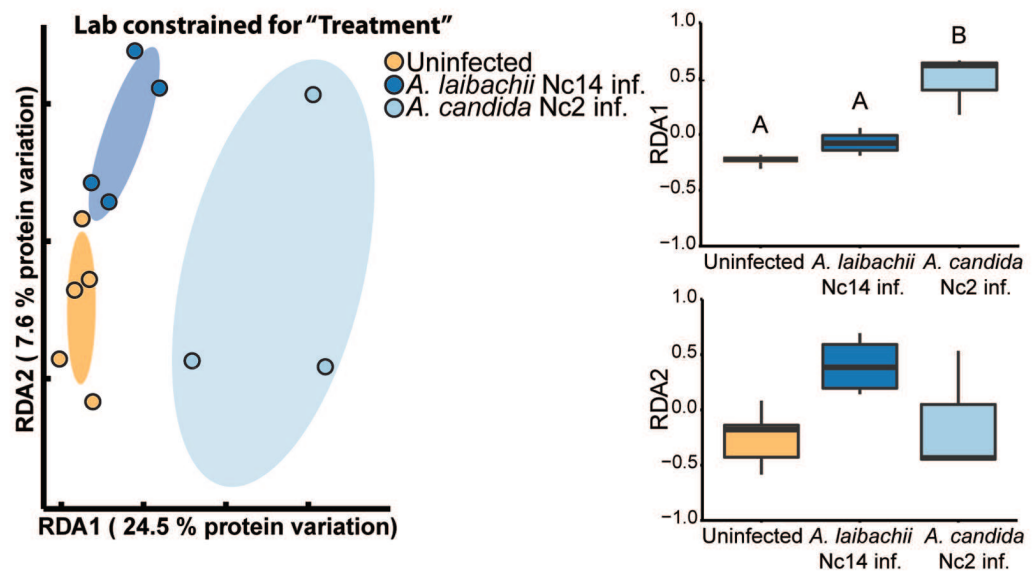

FIGURE 3 | Wild white rust Albugo sp. infections differ from lab infections, but Albugo laibachii does not change the A. thaliana secretome significantly. (A) RDA constrained for factor "treatments" with all infected samples. Wild samples from Pul (dark green, $n=3$ ) and Gey (light green, $n=2$ ) cluster significantly different to laboratory infections of $A$. laibachii Nc14 (dark blue, $n=4$ ) and A. candida Nc2 (light blue, $n=3$ ). Confidence ellipses are based on standard error. (B) RDA constrained for factor "treatments" with all laboratory samples. Uninfected (yellow, $n=5$ ) and A. laibachii Nc14 (dark blue, $n=4)$ infected samples cluster closely together apart from $A$. candida Nc2 (light blue, $n=3$ ) infected samples. The spread of the protein samples on both axes plotted in boxplots indicates a significant difference between $A$. candida Nc2 and A. laibachii Nc14/uninfected samples along RDA1-axis with $24.5 \%$ of the total variation (Tukey honestly significant difference (HSD), $p$-value < 0.05). (C) Arabidopsis thaliana Ws-0 grown in a common garden experiment under wild conditions (red, asymptomatic plants; pink, Albugo-infected plants) has significantly different secretome compositions compared to $A$. thaliana Ws-0 grown under laboratory conditions (yellow, uninfected; orange, Albugo-infected). Confidence ellipses (0.95) are based on standard error. All samples presented in (C) were analyzed with a Q Exactive Plus following in-solution digestion (see liquid chromatography tandem mass spectrometry (LC-MS/MS) Analysis).

Constrained analyses revealed clear differences in protein composition of the infected samples: $63 \%$ of the total protein variation was constrained by distinguishing the samples from Pul, Gey, and the two Albugo lab infections (factor "treatment"; ANOVA, p-value: 0.015; Figure 3A). Pul and Gey infections clustered closely together with significant separation from both A. laibachii Nc14 and A. candida Nc2 infected samples in the laboratory (Figure 3A). Even though the wild samples were from spatially separated sampling sites and infected with different $A$. laibachii strains, there were no significant differences in their secretome composition. Comparable to the apoplastic secretome of uninfected and asymptomatic $A$. thaliana plants (see The Secretome of Wild-Grown A. thaliana Differs Significantly From Lab-Grown Plants), wild plants showing a white rust phenotype differ significantly from plants showing white rust symptoms following infections under controlled lab conditions.

To unravel to which extent Albugo sp. can change the A. thaliana protein secretion following successful colonization, we compared for each environmental condition (laboratory/wild) the secretome of plants showing successful infection of Albugo with uninfected plants. Under laboratory conditions, $32 \%$ of the total variation were constrained by separating uninfected, A. candida Nc2 infected and A. laibachii Nc14 infected samples (ANOVA, p-value: 0.013; Figure 3B). All uninfected/symptomless samples clustered closely together with A. laibachii Nc14 infected leaf samples, apart and with no significant overlap with $A$. candida Nc2 infected samples. 
Considering the spread of the plotted samples along RDA1 ( $x$-axis; explains $24 \%$ of the protein variation) there is a significant difference between the $A$. candida $\mathrm{Nc} 2$ and uninfected/A. laibachii Nc14 infected samples (Tukey HSD, p-value: 0.001; Figure 3B). The uninfected and A. laibachii $\mathrm{Nc} 14$ infected samples cluster closely together without significant differences along this axis and with only weak differences along the $y$-axis. Therefore, under controlled conditions A. laibachii Nc14 infections had non-significant effects on the abundance of secreted $A$. thaliana proteins.

In order to better unravel the environmental influences on Arabidopsis secretomes and to check whether differences between lab and field were due to differences between Ws-0 used in the lab and wild plants, we planted A. thaliana Ws-0 (Albugo susceptible) and Sf-2 (Albugo resistant) plants, in a common garden experiment (described in Agler et al., 2016). Nearly all wild-grown $A$. thaliana Ws-0 plants were infected in spring (mostly by A. laibachii strain MPI1) and we compared these to laboratory experiments with an isolate of this Albugo strain. The high infection rates of Ws-0 impeded sampling of asymptomatic plants. RDA constrained for "treatments" (ANOVA, $p$-value: 0.001) showed a significant difference between the secretomes of field- and lab-grown $A$. thaliana Ws-0. Under laboratory conditions, $A$. thaliana Ws-0 plants infected with $A$. laibachii MPI1 did not significantly differ from uninfected plants. This was comparable to field-grown $A$. thaliana, which showed dense clustering of samples irrespective of infection status (Figure 3C). Albugo-resistant A. thaliana Sf-2 samples clustered closely with $A$. thaliana Ws- 0 . The single replicate from Pul and Gey that was analyzed with the same method clustered away from the plants of the common garden experiment but were more variable. Therefore, these results confirmed differences that were observed between lab-grown plants ((un-)infected) and wild Arabidopsis samples from Pul and Gey (Figures 1 and $\mathbf{3 A}$ ).

Constraining for differences between infected and uninfected samples by site (i.e., within each of Pul and Gey) explained $24 \%$ of the variation in secretome composition, but this was not significant (ANOVA, p-value: 0.744; Supplementary Figure S5A). Similarly, constraining for infection status across both natural sites, which only distinguishes all plants with white rust symptoms from symptomless plants, explained $6 \%$ of the total protein variation and was not significant (ANOVA, p-value: 0.686; Supplementary Figure S5B). This indicates that similar to results in the lab, the protein composition in A. thaliana apoplastic fluid is highly similar in A. laibachiiinfected and -uninfected wild plants (overlapping confidence ellipses based on standard error; Supplementary Figure S5A). Similar to uninfected wild plants (Figure 2), A. laibachiiinfected wild plants exhibited high levels of PR proteins like beta-1, 3-glucanases (PR2 AT3G57260.1, AT3G57240.1), PR1 (AT2G14610.1) and PR5 (AT1G75040.1; APEX $\log _{2}$ abundance $>5.0$; Figure 4). Since there was no significant difference (paired $t$-test, $p$-value $\geq 0.05$ ) in abundance of these proteins in uninfected wild plants compared to wild plants showing white rust symptoms, A. laibachii infection does not significantly affect $A$. thaliana defense protein secretion.
Furthermore, A. laibachii infections do not reduce the secretion of defense proteins that were already present in plants prior to infection. These results suggest that the obligate biotroph A. laibachii is adapted to A. thaliana triggered immune responses in the wild and can complete its infection cycle without severe suppression of the apoplastic protein based defense machinery.

\section{Bacterial Alpha Diversity Changes with Albugo sp. Infection While Fungal Community Compositions Are More Stable}

To unravel which biotic factors might have triggered the observed elevated levels of PR proteins under field conditions regardless of Albugo infection status, we characterized the endophytic microbial communities of Albugo sp. infected versus uninfected A. thaliana plants in Pul and Gey via amplicon sequencing (bacterial 16S rRNA, fungal ITS).

The sampling was done at the same time points as for proteomics and the processing was described in Agler et al. (2016) (see Amplicon Sequencing of Microbial Communities). Unconstrained ordination of the infected and uninfected wild samples indicated that the bacterial communities were fairly variable and clustered by the sampling time point (Figure 5; Supplementary Figure S4). Similar to fungi, bacterial communities grouped by plant generations (the samples that were harvested in December 2013 and March 2014 are one plant generation since $A$. thaliana germinates in fall, is vegetative over winter and dies in late spring following reproduction; Figure 5; Supplementary Figure S4). Contrary to the bacterial communities, only the fungal community in Gey clusters separately from Pul samples, supporting previous results suggesting that fungi are more location-specific than bacteria (Agler et al., 2016; Coleman-Derr et al., 2016). Endophytic fungi, detected at Pul or Gey, belonged largely to the order Pleosporales or the class of Leotiomycetes, which are, often necrotrophic, ascomycotal fungi (Supplementary Figure S3). Calculating the alpha diversity within the bacterial and fungal communities in the infected and uninfected samples revealed a decrease of the diversity in infected plants (Figure 5; Supplementary Figure S4).

Taken together the results indicate that Albugo sp. infections have a stronger influence on the colonization of bacteria where sample clustering is more variable and Albugo-infection status correlates to diversity (Agler et al., 2016). We therefore propose that fungi trigger the observed host immune responses, since these communities are similar in infected and uninfected samples and therefore seem to be less under the control of Albugo.

\section{Albugo laibachii Infections Are Less Affected by Altered Host Hormone Levels than $\mathrm{Hpa}$}

Arabidopsis thaliana immune responses to biotic stresses often result in alteration of plant hormone levels including SAmediated defense against (hemi-)biotrophs or jasmonic acid 


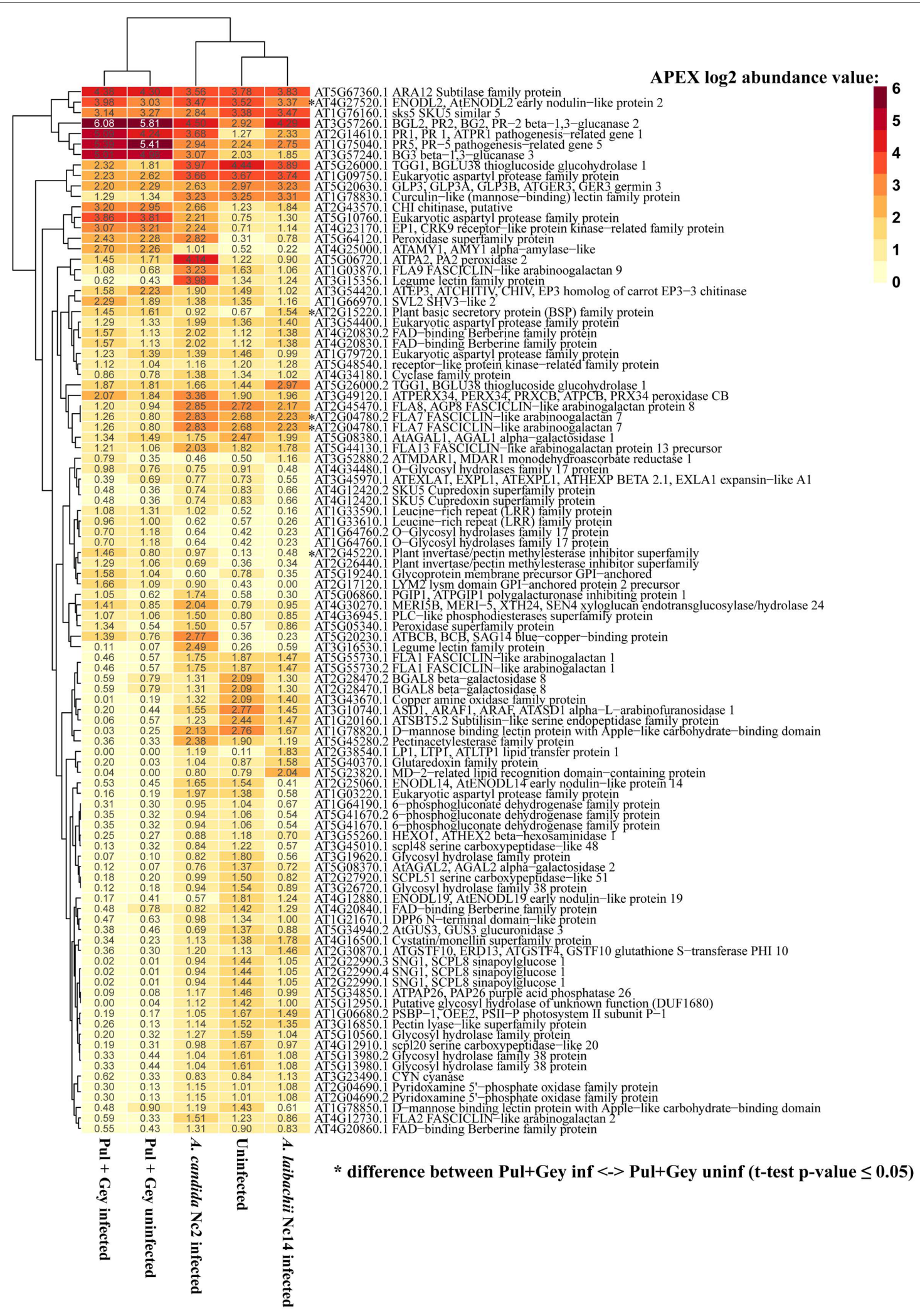

FIGURE 4 | Asymptomatic, uninfected wild $A$. thaliana plants show an activated immune system, which is not changed by Albugo sp. infections. The most abundant $A$. thaliana proteins in wild Albugo sp.-infected and -uninfected plants are related to defense responses. APEX abundance values were averaged across replicates and $\log _{2}$ transformed. Significant differences between wild samples are indicated by symbols. 


\section{Bray-Curtis Dist. PCoA}

\section{Alpha Diversity}
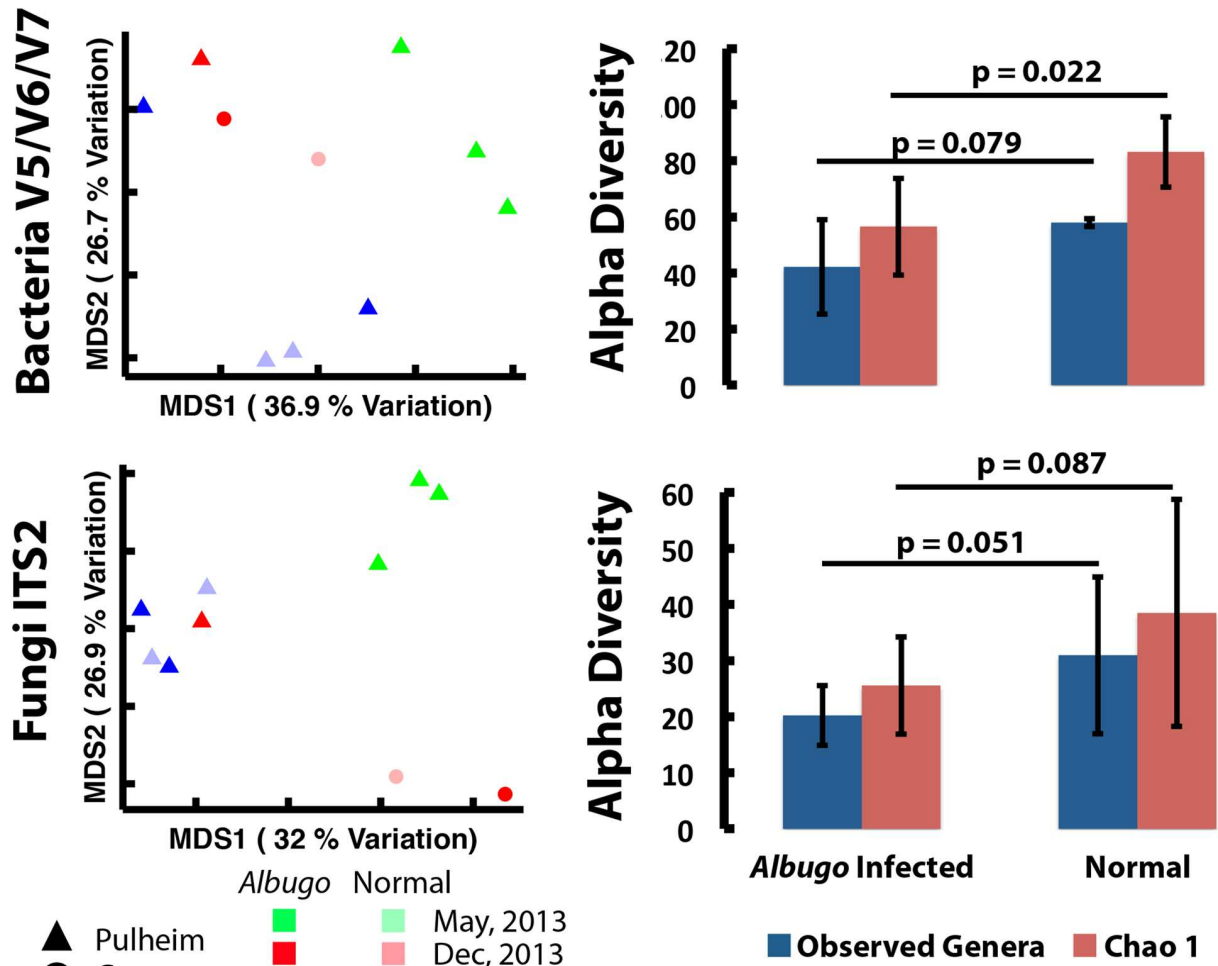

FIGURE 5 | Unconstrained ordination of bacterial and fungal communities and their respective alpha diversity. Principle component analyses of microbial communities from Pul and Gey, based on amplicon sequencing (bacterial 16S rRNA V5/N6/V7 region, fungal ITS2 region). The alpha diversity within bacterial and fungal communities decreases in infected plants.

(JA)/ethylene-mediated defense against necrotrophs (Thomma et al., 1998; Glazebrook, 2005). Especially PR1, PR2, and PR5 proteins were shown to be SA responsive (Uknes et al., 1992), and we showed that they were highly abundant in all wild $A$. thaliana samples. Hpa has an overlapping host range and is a natural competitor of Albugo sp. on A. thaliana, because as obligate biotrophic oomycetes they occupy a similar niche.

During our experimentation, we phenotypically observed far more A. laibachii infection in the wild than other biotrophic pathogens of $A$. thaliana like Hpa. Therefore, we wanted to know if $A$. laibachii is better adapted to the primed immune system (i.e., constantly high PR protein levels) than Hpa. We compared both pathogens in growth assays on $A$. thaliana hormone mutants for their infection efficiency (Figure 6). Oomycete growth quantification via qPCR showed that $\mathrm{Hpa}$ Noco2 is especially affected in ABA biosynthesis-deficient mutants (aba31, aba2-12, negative effect), an SA-induction deficient mutant (sid2-2, positive effect) and a mutant with constitutive expression of PR-genes (cpr5, positive effect) compared to A. laibachii. Furthermore, $\mathrm{Hpa}$ Noco2 growth is significantly lowered in the ein2-1 mutant (ethylene insensitive), which has elevated JA levels after pathogen treatment and constitutive $P R 1$ expression (Penninckx et al., 1998; Chen et al., 2009). Two different A. laibachii strains (MPI1 and Nc14) were tested. Contrary to Hpa, A. laibachii Nc14 and MPI1 colonization was very resilient, since its growth was only affected (relative to mock control) in the cpr5 (Nc14/MPI1) and the aba2-12 (MPI1) mutant backgrounds. In total, $\mathrm{Hpa}$ Noco 2 growth varied more in the different mutant backgrounds (significant difference to mock control in five of eight mutants), than A. laibachii (significant difference in one of eight mutants for $\mathrm{Nc14}$ and two of eight mutants for MPI1). This suggests that A. laibachii strains show a high plasticity to adapt to a broad range of host pre-existing defense fluctuations. This plasticity might give an advantage in competing for limited growth space in nature.

\section{DISCUSSION}

Arabidopsis thaliana is the best-studied flowering plant under controlled lab conditions (Koornneef and Meinke, 2010). How A. thaliana physiology changes and what happens on a molecular level under natural conditions compared to lab conditions is largely unknown. We have addressed this knowledge gap by comparing the apoplastic secretome of $A$. thaliana plants grown under standard experimental conditions in the lab with wild plants in stable, well-established, populations or with wild-grown 


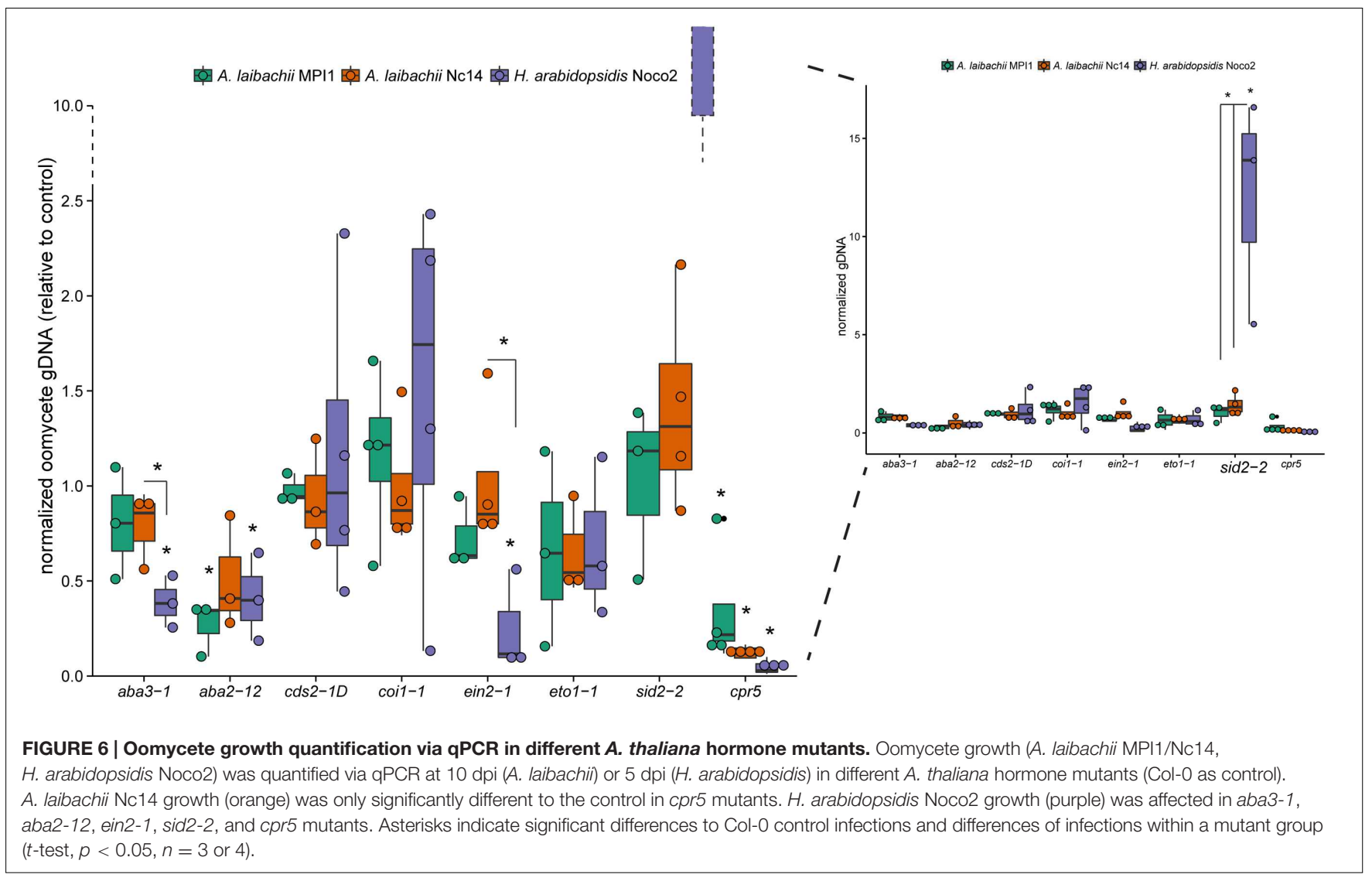

plants in a common garden experiment. Our data demonstrates that morphologically healthy, wild- or field-grown A. thaliana plants are significantly different in physiology from plants grown under controlled lab conditions. The main difference is that naturally grown plants have significantly higher abundances of defense- and stress-related proteins in the apoplastic space (Figure 2).

\section{Asymptomatic Wild $A$. thaliana Has an Induced Immune System}

The apoplastic space is important for plant defense and pathogen virulence, as it is one of the main contact points of the host to invading pathogens (e.g., Kaffarnik et al., 2009; Floerl et al., 2012; Ali et al., 2014; Kim et al., 2014) and beneficial endophytes (e.g., Dong et al., 1994; Chi et al., 2005). Colonizing pathogens are generally recognized by plant cells in the apoplast where they are directly attacked by plant defenses. Upon microbe recognition, plants try to limit their growth by, e.g., reacting with a burst of reactive oxygen species (ROS; Daudi et al., 2012) or producing antimicrobial proteins from the PR-family (PR proteins; Loon et al., 2006). In apoplastic fluids extracted from wild, asymptomatic A. thaliana plants we found the most abundant proteins were PR2 (pathogenesis-related 2), PR5, BG3 (beta-1,3-glucanase 3), PR1 and eukaryotic aspartyl protease, which indicates triggered immune responses. Especially PR1, PR2, and PR5 are known marker proteins for SA-dependent systemic acquired resistance (SAR; Uknes et al., 1992, 1993), which is a long-lasting form of broad-spectrum disease resistance against avirulent pathogens in the whole plant (Maleck et al., 2000). High abundance of PR1 and SAR often goes along with redox regulation and accumulation of ROS, which are generated by nicotinamide adenine dinucleotide phosphate (NADPH) oxidases or apoplastic peroxidases (Mammarella et al., 2015). A. thaliana encodes several peroxidases, of which the apoplastic peroxidase 34 (PRX34, AT3G49120.1) is specifically activated under plant defense conditions, as well as peroxidase 33 (PRX33, AT3G49110.1; Daudi et al., 2012; Mammarella et al., 2015). However, PRX34 and PRX33 peroxidases were not significantly different in abundance in lab or wild plants and PRX33 was generally only very low abundant (not among top 100 proteins, Figure 4). A further 16 extracellular class III peroxidases that were shown to be expressed in leaves (Welinder et al., 2002), were either not detected or only at very low abundances with no difference between symptomless wild and lab samples. This could suggest that no ROS-burst or hypersensitive response was triggered in asymptomatic wild plants. In labgrown unchallenged plants, only low levels of PR-proteins were detectable, consistent with the basal level that has been described for A. thaliana in previous studies (Floerl et al., 2012; Trentin et al., 2015).

The most abundant protein in untreated lab grown samples is a thioglucoside glucohydrolase (known as myrosinase; AT5G26000.1), which is significantly more abundant compared 


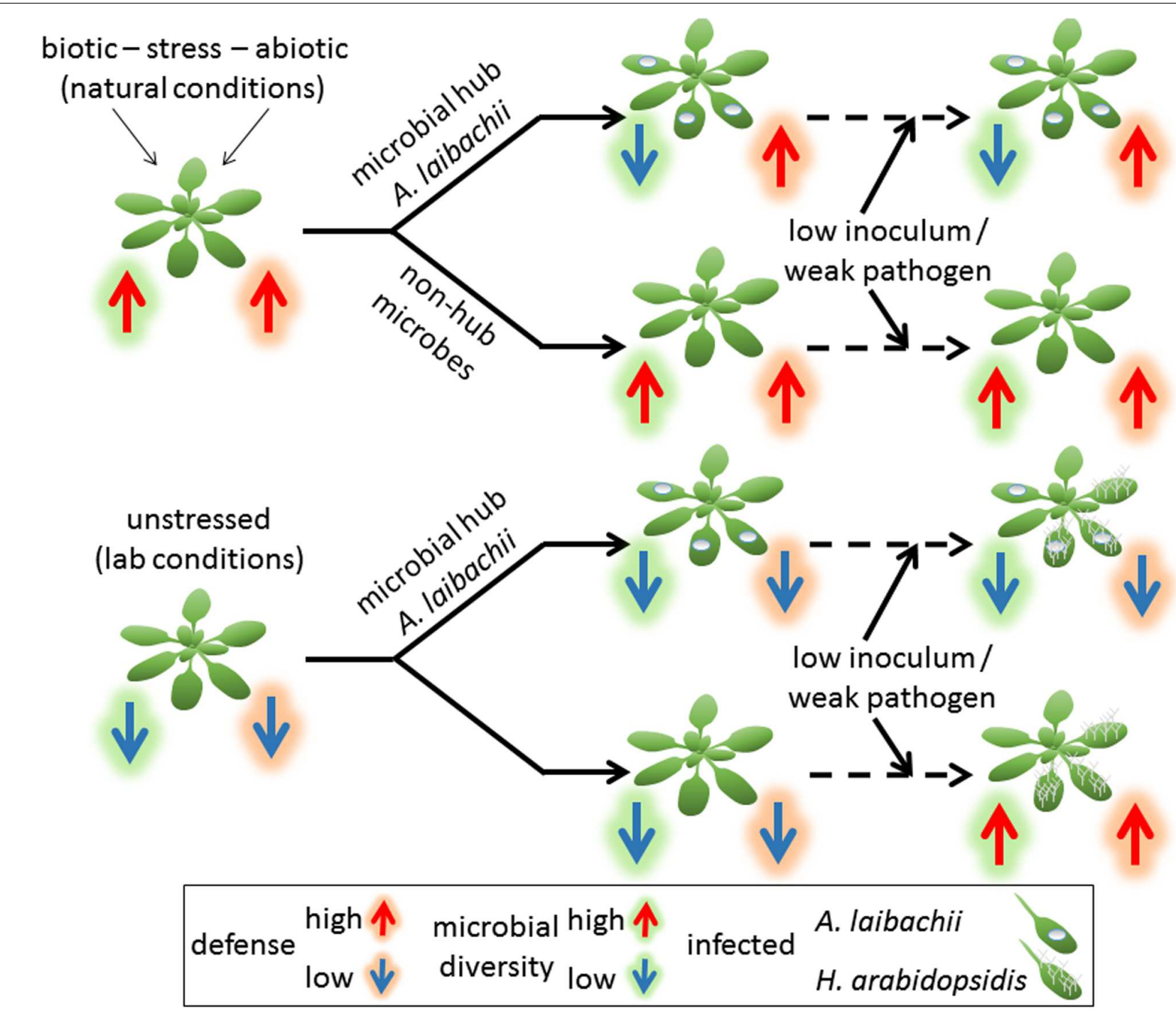

FIGURE 7 | The well-adapted microbial hub Albugo keeps host immunity active to gain colonization advantages over competitors in nature. Under natural growth conditions, $A$. thaliana shows an induced immune system and a high microbial diversity in the phyllosphere. Infections of $A$. laibachii influence the microbial community structure, but do not suppress host defense reactions so that colonization of weak pathogens is hindered. If the $A$. thaliana immune system is not induced, as in plants grown under lab conditions, pathogens like weak strains of $H$. arabidopsidis can infect $A$. thaliana even after a preceding Albugo infection. Albugo does not trigger strong host defense reactions, which allows the growth of $H$. arabidopsidis.

to asymptomatic field samples (Figure 2). Glucosinolates are secondary metabolites that can be cleaved by the enzyme thioglucoside glucohydrolase resulting in toxic products against fungi and insects (Barth and Jander, 2006; Halkier and Gershenzon, 2006; Bednarek et al., 2009). As such it can deter generalist herbivores, but might attract crucifer specialists (Barth and Jander, 2006). Taking lab-uninfected A. thaliana protein abundances as a measure for protein levels under unstressed conditions, we hypothesize that microbes colonizing healthy, uninfected wild plants lead to suppression of myrosinases and glucosinolates in nature. Thus, defense against eukaryotic microbes might be lowered, facilitating the colonization of fungi and oomycetes in nature and ultimately leading to the induced immune system.

\section{Restructuring the Leaf Microbial} Community by the Microbial Hub A. laibachii Is Not Mediated via Host Protein Secretion

Naturally grown $A$. thaliana plants accommodate a broad range of microbes (Vorholt, 2012; Agler et al., 2016), which are probably responsible for the observed activated defense and myrosinase suppression. Thus, our goal was to dissect the endophytic microbial community to identify responsible key organisms. Our community profiling revealed that bacteria did not differ markedly between the two wild populations of Pul and Gey, whereas fungal communities showed a significant location specificity. This indicates a rather homogenous spread 
of bacterial inoculum, while fungal dispersal/growth is more unequal and site specific. This agrees with previous reports that bacterial communities vary in their relative abundance of species between sites, while fungal communities differ by presence/absence between different sites (Agler et al., 2016; Coleman-Derr et al., 2016). Bacterial and fungal communities were fairly stable over one plant generation (December 2013, March 2014) even though challenged by natural biotic and abiotic fluctuations, but differed significantly from one to the next plant generation (May 2013).

Endophytic fungi belonged largely to the order Pleosporales, which has many members that are necrotrophic plant pathogens like the detected Alternaria sp., Ascochyta sp. or Boeremia sp. Even though these fungi were detected as endophytes via sequencing, the plants did not show any signs of necrosis and were otherwise healthy during sampling. Possibly, these fungi initially colonized plants and triggered the observed immune responses and the primed host immune system restricted their growth. Although Alternaria brassicicola was shown to trigger SA-marker gene expression, growth restriction, and resistance against $A$. brassicicola relies on callose-deposition (Ton and Mauch-Mani, 2004). Even though we did not observe the callose synthase enzymes (e.g., AtGSL5), we cannot exclude their activation, since they are cellular and not detectable in the apoplast.

Besides fungi, the most abundant eukaryotic microbes observed in wild $A$. thaliana populations were the causal agents of white rust symptoms, Albugo sp. Endophytic bacterial communities of wild $A$. thaliana plants changed after infection by Albugo sp. showing significantly reduced alpha diversity and Gammaproteobacteria (mostly Pseudomonas sp.) dominating bacterial communities. Again, this is consistent with Agler et al. (2016), which highlighted an A. laibachii-mediated reduction of the bacterial alpha diversity of endo- and epiphytes and a stabilization of the community structure. Identifying A. laibachii as a hub organism structuring the A. thaliana microbial community left one main question: Does the hub reduce bacterial diversity via triggering plant defense or is there a direct interaction between hub and microbes? With this work, we can now show that the increase of relative abundance of major bacterial taxa and limiting the bacterial diversity is not mediated via the host protein secretion, as the hub microbe $A$. laibachii does not influence the $A$. thaliana protein secretion significantly. This suggests that direct microbemicrobe interactions might take place in the apoplastic space that result in observed decreased diversity of bacteria. Investigating the secretome of Albugo sp. during apoplastic space colonization could better resolve such microbe-microbe interactions.

\section{Albugo laibachii Tolerates Apoplastic Broad-Spectrum Immune Responses Instead of Suppressing Them}

Even though $A$. laibachii was shown to suppress resistancegene-mediated broad-spectrum resistance (non-systemic; Cooper et al., 2008), we showed that this does not translate into broad effects on suppressing apoplastic defense. Thus, Albugo sp. are still able to go through their whole infection cycle without suppressing already activated host immune responses in the apoplast. In controlled lab conditions, where replicability was significantly higher, revealed that A. laibachii Nc14-infections lowered the protein abundance of only four A. thaliana proteins (serine carboxypeptidase-like protein, pectinacetylesterase family protein, mannose binding lectin protein and alpha-galactosidase; Supplementary Figure S8). Two of these proteins, pectinacetylesterase and alpha-galactosidase, could be involved in cell wall organization processes and could therefore be A. laibachii target proteins for haustoria formation in the host cells. The other two proteins, serine carboxypeptidase and mannose binding lectin protein, might contribute to plant defense reactions as carboxypeptidases are involved in protein degradation and many lectins bind foreign polysaccharides and can play a role in pathogen recognition (Lehfeldt et al., 2000; Sharon and Lis, 2004). Nevertheless, A. laibachii has suppressive effects on the abundance of only $4 \%$ of the host secreted proteins, none of which are classics of defense, like PR proteins or peroxidases (Supplementary Figure S8). Therefore, one might speculate that instead of broad-spectrum suppression, Albugo must have developed some mechanisms to protect its hyphae from immune responses. Similar mechanisms have been observed for fungal pathogens that use $\alpha$-1-3-glucan to protect chitin in the cell wall from degradation by plant chitinases (Oliveira-Garcia and Valent, 2015).

Being adapted to the naturally occurring immune responses in wild host plants and not triggering further defense reactions seems to be advantageous in competing for limited growth space. A. laibachii appears to not only tolerate host-mediated broadspectrum immune responses but also its growth is very resilient in host plants with altered hormone levels. On the other hand, the potential competitor Hpa - a common obligate biotroph pathogen on A. thaliana in the wild (Holub et al., 1994) is strongly affected (positively and negatively) by hormonal changes. The cpr5 mutant secretome might be most similar to wild $A$. thaliana plants as it has high protein concentrations of PR1, PR2, PR5 and the defensin protein PDF1.2 (Bowling et al., 1997). The cpr5 mutant also harbors high levels of SA compared to Col-0 wild type plants and was shown to be fully resistant against $\mathrm{Hpa}$ Noco2 (Bowling et al., 1997; Clarke et al., 2000). Infection screens were formerly based on conidiophores or spore counts at $7 \mathrm{dpi}$ (Bowling et al., 1997; Clarke et al., 2000). We also did not observe sporulation after $\mathrm{Hpa}$ Noco2 infections at $5 \mathrm{dpi}$, but the more sensitive qPCR quantification revealed low levels of Hpa growth (Figure 6). A. laibachii infections were routinely harvested at 10 dpi and showed weak white rust sporulation in cpr5 mutants, as well as stronger quantified growth than $H p a$, highlighting the capability of $A$. laibachii to grow even under induced plant defense. Furthermore, Hpa infections were affected in aba2-12, aba3-1, ein2-1, and sid2-2 mutant background while A. laibachii was not. The sid2-2 mutant, defective in ISOCHORISMATE SYNTHASE 1, does not accumulate pathogen-inducible SA and is impaired in its SAR activation (Nawrath and Métraux, 1999; Bernsdorff et al., 2015). Thus the sid2-2 mutant is hyper susceptible to Hpa infections, whereas A. laibachii grew only 
slightly better on these plants (Figure 6; Nawrath and Métraux, 1999). The mutants that are impaired in ABA biosynthesis (aba31, aba2-12) did not affect A. laibachii Nc14 growth (A. laibachii MPI1 was affected in aba2-12), but $\mathrm{Hpa}$ Noco2 grew significantly less in all of these (Figure 6; Léon-Kloosterziel et al., 1996; Adie et al., 2007). The impairment of ABA synthesis results in decreased levels of JA and increased levels of SA-induced genes after pathogen infection, compared to wild type (Adie et al., 2007). Together, our results imply different strategies of the two pathogens: Hpa can quickly take advantage of suppressed defense where it probably would outcompete A. laibachii, but it is also more susceptible to plant defenses than the highly plastic and robust Albugo. Probably the latter situation is more common in many plant populations, explaining our observation of less $H p a$ in the wild.

In summary, our work gives significant new experimental insight into how plants behave under natural conditions on a molecular level and dissects clear, significant differences to experiments under controlled lab conditions (Figure 7). It further indicates that oomycete pathogens of the genus Albugo, which are important microbial hubs regulating the A. thaliana microbial community in the wild, are extremely fine-tuned to neither trigger strong host defense reactions, nor to act on broadspectrum defense suppression in the apoplast. Therefore, we hypothesize that Albugo is well adapted to an active host immune system, which gives them an advantage over competitors in fighting for limited growth space in the same niche.

\section{REFERENCES}

Adie, B. A. T., Pérez-Pérez, J., Pérez-Pérez, M. M., Godoy, M., Sánchez-Serrano, J.J., Schmelz, E. A., et al. (2007). ABA is an essential signal for plant resistance to pathogens affecting JA biosynthesis and the activation of defenses in Arabidopsis. Plant Cell 19, 1665-1681. doi: 10.1105/tpc.106.048041

Agler, M. T., Ruhe, J., Kroll, S., Morhenn, C., Kim, S.-T., Weigel, D., et al. (2016). Microbial hub taxa link host and abiotic factors to plant microbiome variation. PLoS Biol. 14:e1002352. doi: 10.1371/journal.pbio.1002352

Ali, A., Alexandersson, E., Sandin, M., Resjö, S., Lenman, M., Hedley, P., et al. (2014). Quantitative proteomics and transcriptomics of potato in response to Phytophthora infestans in compatible and incompatible interactions. BMC Genomics 15:497. doi: 10.1186/1471-2164-15-497

Amrine, K. C. H., Blanco-Ulate, B., and Cantu, D. (2015). Discovery of core biotic stress responsive genes in Arabidopsis by weighted gene co-expression network analysis. PLoS ONE 10:e0118731. doi: 10.1371/journal.pone.0118731

Asai, S., Rallapalli, G., Piquerez, S. J. M., Caillaud, M.-C., Furzer, O. J., Ishaque, N., et al. (2014). Expression profiling during Arabidopsis/downy mildew interaction reveals a highly-expressed effector that attenuates responses to salicylic acid. PLoS Pathog. 10:e1004443. doi: 10.1371/journal.ppat.1004443

Barth, C., and Jander, G. (2006). Arabidopsis myrosinases TGG1 and TGG2 have redundant function in glucosinolate breakdown and insect defense. Plant J. 46, 549-562. doi: 10.1111/j.1365-313X.2006.02716.x

Bednarek, P., Piślewska-Bednarek, M., Svatoš, A., Schneider, B., Doubský, J., Mansurova, M., et al. (2009). A glucosinolate metabolism pathway in living plant cells mediates broad-spectrum antifungal defense. Science 323, 101-106. doi: 10.1126/science.1163732

Berardini, T. Z., Mundodi, S., Reiser, L., Huala, E., Garcia-Hernandez, M., Zhang, P., et al. (2004). Functional annotation of the Arabidopsis genome using controlled vocabularies. Plant Physiol. 135, 745-755. doi: 10.1104/ pp.104.040071

Bernsdorff, F., Döering, A.-C., Gruner, K., Schuck, S., Bräutigam, A., and Zeier, J. (2015). Pipecolic acid orchestrates plant systemic acquired resistance and

\section{AUTHOR CONTRIBUTIONS}

Experiments were conceived and designed by JR, MA, IF, and EK. The experiments were performed by JR, MA, AP, KK, and IF. Data analysis was conducted by JR and MA. The manuscript was written by JR and EK. All authors corrected the manuscript and discussed the data.

\section{FUNDING}

We acknowledge funding by the Max Planck Society and the Cluster of Excellence on Plant Science (CEPLAS).

\section{ACKNOWLEDGMENT}

The authors would like to thank Jaqueline Bautor for help with Hpa infections, and Thomas Colby and Anne Harzen for their excellent technical support in proteomics experiments.

\section{SUPPLEMENTARY MATERIAL}

The Supplementary Material for this article can be found online at: http://journal.frontiersin.org/article/10.3389/fpls.2016. 00820

defense priming via salicylic acid-dependent and -independent pathways. Plant Cell 28, 102-129. doi: 10.1105/tpc.15.00496

Bowling, S. A., Clarke, J. D., Liu, Y., Klessig, D. F., and Dong, X. (1997). The cpr5 mutant of Arabidopsis expresses both NPR1-dependent and NPR1-independent resistance. Plant Cell 9, 1573-1584. doi: 10.1105/tpc.9.9.1573

Braisted, J., Kuntumalla, S., Vogel, C., Marcotte, E., Rodrigues, A., Wang, R., et al. (2008). The APEX quantitative proteomics tool: generating protein quantitation estimates from LC-MS/MS proteomics results. BMC Bioinformat. 9:529. doi: 10.1186/1471-2105-9-529

Caillaud, M. C., Asai, S., Rallapalli, G., Piquerez, S., Fabro, G., and Jones, J. D. (2013). A downy mildew effector attenuates salicylic acid-triggered immunity in Arabidopsis by interacting with the host mediator complex. PLoS Biol. 11:e1001732. doi: 10.1371/journal.pbio.1001732

Chen, H., Xue, L., Chintamanani, S., Germain, H., Lin, H., Cui, H., et al. (2009). ETHYLENE INSENSITIVE3 and ETHYLENE INSENSITIVE3-LIKE1 Repress SALICYLIC ACID INDUCTION DEFICIENT2 expression to negatively regulate plant innate immunity in Arabidopsis. Plant Cell 21, 2527-2540. doi: 10.1105/tpc.108.065193

Chi, F., Shen, S.-H., Cheng, H.-P., Jing, Y.-X., Yanni, Y. G., and Dazzo, F. B. (2005). Ascending migration of endophytic rhizobia, from roots to leaves, inside rice plants and assessment of benefits to rice growth physiology. Appl. Environ. Microbiol. 71, 7271-7278. doi: 10.1128/AEM.71.11.7271-7278.2005

Clarke, J. D., Volko, S. M., Ledford, H., Ausubel, F. M., and Dong, X. (2000). Roles of Salicylic acid, jasmonic acid, and ethylene in cpr-induced resistance in Arabidopsis. Plant Cell 12, 2175-2190. doi: 10.2307/3871113

Coleman-Derr, D., Desgarennes, D., Fonseca-Garcia, C., Gross, S., Clingenpeel, S., Woyke, T., et al. (2016). Plant compartment and biogeography affect microbiome composition in cultivated and native Agave species. New Phytol. 209, 798-811. doi: 10.1111/nph.13697

Cooper, A. J., Latunde-Dada, A. O., Woods-Tör, A., Lynn, J., Lucas, J. A., Crute, I. R., et al. (2008). Basic Compatibility of Albugo candida in Arabidopsis thaliana and Brassica juncea causes broad-spectrum suppression of innate immunity. Mol. Plant Microbe Interact. 21, 745-756. doi: 10.1094/MPMI-21-6-0745 
Cox, J., Hein, M. Y., Luber, C. A., Paron, I., Nagaraj, N., and Mann, M. (2014). Accurate proteome-wide label-free quantification by delayed normalization and maximal peptide ratio extraction, termed MaxLFQ. Mol. Cell. Proteomics 13, 2513-2526. doi: 10.1074/mcp.M113.031591

Cox, J., and Mann, M. (2008). MaxQuant enables high peptide identification rates, individualized p.p.b.-range mass accuracies and proteome-wide protein quantification. Nat. Biotechnol. 26, 1367-1372. doi: 10.1038/nbt.1511

Cox, J., and Mann, M. (2012). 1D and 2D annotation enrichment: a statistical method integrating quantitative proteomics with complementary highthroughput data. BMC Bioinforma. 13(Suppl. 16):S12. doi: 10.1186/1471-210513-S16-S12

Craig, R., and Beavis, R. C. (2004). TANDEM: matching proteins with tandem mass spectra. Bioinformatics 20, 1466-1467. doi: 10.1093/bioinformatics/bth092

Daudi, A., Cheng, Z., O’brien, J. A., Mammarella, N., Khan, S., Ausubel, F. M., et al. (2012). The apoplastic oxidative burst peroxidase in Arabidopsis is a major component of pattern-triggered immunity. Plant Cell 24, 275-287. doi: 10.1105/tpc.111.093039

De Vos, M., Van Oosten, V. R., Van Poecke, R. M. P., Van Pelt, J. A., Pozo, M. J., Mueller, M. J., et al. (2005). Signal signature and transcriptome changes of arabidopsis during pathogen and insect attack. Mol. Plant Microbe Interact. 18, 923-937. doi: 10.1094/MPMI-18-0923

De Wit, P. J. G. M., Mehrabi, R., Van Den Burg, H. A., and Stergiopoulos, I. (2009). Fungal effector proteins: past, present and future. Mol. Plant Pathol. 10, 735-747. doi: 10.1111/j.1364-3703.2009.00591.x

Delaunois, B., Jeandet, P., Clément, C., Baillieul, F., Dorey, S., and Cordelier, S. (2014). Uncovering plant-pathogen crosstalk through apoplastic proteomic studies. Front. Plant Sci. 5:249. doi: 10.3389/fpls.2014.00249

Deutsch, E. W., Mendoza, L., Shteynberg, D., Farrah, T., Lam, H., Tasman, N., et al. (2010). A guided tour of the trans-proteomic pipeline. Proteomics 10, 1150-1159. doi: 10.1002/pmic.200900375

Doehlemann, G., and Hemetsberger, C. (2013). Apoplastic immunity and its suppression by filamentous plant pathogens. New Phytol. 198, 1001-1016. doi: 10.1111/nph.12277

Dong, Z., Canny, M. J., Mccully, M. E., Roboredo, M. R., Cabadilla, C. F., Ortega, E., et al. (1994). A nitrogen-fixing endophyte of sugarcane stems (A New Role for the Apoplast). Plant Physiol. 105, 1139-1147.

Emanuelsson, O., Nielsen, H., Brunak, S., and Von Heijne, G. (2000). Predicting subcellular localization of proteins based on their N-terminal amino acid sequence. J. Mol. Biol. 300, 1005-1016. doi: 10.1006/jmbi.2000.3903

Floerl, S., Druebert, C., Majcherczyk, A., Karlovsky, P., Kües, U., and Polle, A. (2008). Defence reactions in the apoplastic proteome of oilseed rape (Brassica napus var. napus) attenuate Verticillium longisporum growth but not disease symptoms. BMC Plant Biol. 8:129. doi: 10.1186/1471-2229-8-129

Floerl, S., Majcherczyk, A., Possienke, M., Feussner, K., Tappe, H., Gatz, C., et al. (2012). Verticillium longisporum infection affects the leaf apoplastic proteome, metabolome, and cell wall properties in Arabidopsis thaliana. PLoS ONE 7:e31435. doi: 10.1371/journal.pone.0031435

Fowler, S., and Thomashow, M. F. (2002). Arabidopsis transcriptome profiling indicates that multiple regulatory pathways are activated during cold acclimation in addition to the cbf cold response pathway. Plant Cell 14, 1675-1690. doi: 10.1105/tpc.003483

Glazebrook, J. (2005). Contrasting mechanisms of defense against biotrophic and necrotrophic pathogens. Annu. Rev. Phytopathol. 43, 205-227. doi: 10.1146/annurev.phyto.43.040204.135923

Halkier, B. A., and Gershenzon, J. (2006). Biology and biochemistry of glucosinolates. Annu. Rev. Plant Biol. 57, 303-333. doi: 10.1146/annurev. arplant.57.032905.105228

Holub, E., Beynon, J., and Crute, I. (1994). Phenotypic and genotypic characterization of interactions between isolates of Peronospora parasitica and accessions of Arabidopsis thaliana. Mol. Plant Microbe Interact. 7, 223-239. doi: 10.1094/MPMI-7-0223

Irieda, H., Maeda, H., Akiyama, K., Hagiwara, A., Saitoh, H., Uemura, A., et al. (2014). Colletotrichum orbiculare secretes virulence effectors to a biotrophic interface at the primary hyphal neck via exocytosis coupled with SEC22mediated traffic. Plant Cell 26, 2265-2281.

Kaffarnik, F. A. R., Jones, A. M. E., Rathjen, J. P., and Peck, S. C. (2009). Effector Proteins of the bacterial pathogen Pseudomonas syringae alter the extracellular proteome of the host plant, Arabidopsis thaliana. Mol. Cell. Proteomics 8, 145-156. doi: 10.1074/mcp.M800043MCP200

Kemen, E., Gardiner, A., Schultz-Larsen, T., Kemen, A. C., Balmuth, A. L., RobertSeilaniantz, A., et al. (2011). Gene gain and loss during evolution of obligate parasitism in the white rust pathogen of Arabidopsis thaliana. PLoS Biol. 9:e1001094. doi: 10.1371/journal.pbio.1001094

Kemen, E., and Jones, J. D. G. (2012). Obligate biotroph parasitism: can we link genomes to lifestyles? Trends Plant Sci. 17, 448-457. doi: 10.1016/j.tplants.2012.04.005

Kemen, E., Kemen, A. C., Rafiqi, M., Hempel, U., Mendgen, K., Hahn, M., et al. (2005). Identification of a protein from rust fungi transferred from haustoria into infected plant cells. Mol. Plant Microbe Interact. 18, 1130-1139. doi: 10.1094/MPMI-18-1130

Kim, J. Y., Wu, J., Kwon, S. J., Oh, H., Lee, S. E., Kim, S. G., et al. (2014). Proteomics of rice and Cochliobolus miyabeanus fungal interaction: insight into proteins at intracellular and extracellular spaces. Proteomics 14, 2307-2318. doi: 10.1002/pmic.201400066

Koornneef, M., and Meinke, D. (2010). The development of Arabidopsis as a model plant. Plant J. 61, 909-921. doi: 10.1111/j.1365-313X.2009.04086.x

Kreps, J. A., Wu, Y., Chang, H.-S., Zhu, T., Wang, X., and Harper, J. F. (2002). Transcriptome changes for Arabidopsis in response to salt, osmotic, and cold stress. Plant Physiol. 130, 2129-2141. doi: 10.1104/pp.008532

Laemmli, U. K. (1970). Cleavage of structural proteins during the assembly of the head of bacteriophage T4. Nature 227, 680-685. doi: 10.1038/227680a0

Lehfeldt, C., Shirley, A. M., Meyer, K., Ruegger, M. O., Cusumano, J. C., Viitanen, P. V., et al. (2000). Cloning of the SNG1 gene of Arabidopsis reveals a role for a serine carboxypeptidase-like protein as an acyltransferase in secondary metabolism. Plant Cell 12, 1295-1306. doi: 10.2307/3871130

Léon-Kloosterziel, K. M., Gil, M. A., Ruijs, G. J., Jacobsen, S. E., Olszewski, N. E., Schwartz, S. H., et al. (1996). Isolation and characterization of abscisic acid-deficient Arabidopsis mutants at two new loci. Plant J. 10, 655-661. doi: 10.1046/j.1365-313X.1996.10040655.X

Lewis, L. A., Polanski, K., De Torres-Zabala, M., Jayaraman, S., Bowden, L., Moore, J., et al. (2015). Transcriptional dynamics driving MAMP-triggered immunity and pathogen effector-mediated immunosuppression in Arabidopsis leaves following infection with Pseudomonas syringae pv tomato DC3000. Plant Cell 27, 3038-3064. doi: 10.1105/tpc.15.00471

Lian, X., Wang, S., Zhang, J., Feng, Q., Zhang, L., Fan, D., et al. (2006). Expression Profiles of 10,422 Genes at Early Stage of Low Nitrogen Stress in Rice Assayed using a cDNA Microarray. Plant Mol. Biol. 60, 617-631. doi: 10.1007/s11103005-5441-7

Loon, L. C. V., Rep, M., and Pieterse, C. M. J. (2006). Significance of inducible defense-related proteins in infected plants. Annu. Rev. Phytopathol. 44, 135162. doi: 10.1146/annurev.phyto.44.070505.143425

Maleck, K., Levine, A., Eulgem, T., Morgan, A., Schmid, J., Lawton, K. A., et al. (2000). The transcriptome of Arabidopsis thaliana during systemic acquired resistance. Nat. Genet. 26, 403-410. doi: 10.1038/82521

Mammarella, N. D., Cheng, Z., Fu, Z. Q., Daudi, A., Bolwell, G. P., Dong, X., et al. (2015). Apoplastic peroxidases are required for salicylic acid-mediated defense against Pseudomonas syringae. Phytochemistry 112, 110-121. doi: 10.1016/j.phytochem.2014.07.010

McKinney, E. C., Ali, N., Traut, A., Feldmann, K. A., Belostotsky, D. A., Mcdowell, J. M., et al. (1995). Sequence-based identification of T-DNA insertion mutations in Arabidopsis: actin mutants act2-1 and act4-1. Plant J. 8, 613-622. doi: 10.1046/j.1365-313X.1995.8040613.x

McMullan, M., Gardiner, A., Bailey, K., Kemen, E., Ward, B. J., Cevik, V., et al. (2015). Evidence for suppression of immunity as a driver for genomic introgressions and host range expansion in races of Albugo candida, a generalist parasite. eLife 4:e04550. doi: 10.7554/eLife.04550

Mittler, R. (2006). Abiotic stress, the field environment and stress combination. Trends Plant Sci. 11, 15-19. doi: 10.1016/j.tplants.2005.11.002

Nagano, A. J., Sato, Y., Mihara, M., Antonio, B. A., Motoyama, R., Itoh, H., et al. (2012). Deciphering and prediction of transcriptome dynamics under fluctuating field conditions. Cell 151, 1358-1369. doi: 10.1016/j.cell.2012.10.048

Nawrath, C., and Métraux, J. P. (1999). Salicylic acid induction-deficient mutants of Arabidopsis express PR-2 and PR-5 and accumulate high levels of camalexin after pathogen inoculation. Plant Cell 11, 1393-1404. doi: 10.2307/ 3870970 
Oksanen, J., Blanchet, F. G., Kindt, R., Legendre, P., Minchin, P. R., O’Hara, R. B., et al. (2015). vegan: Community Ecology Package. R Package Version 2.2-1. Available at: http://CRAN.R-project.org/package = vegan

Oliveira-Garcia, E., and Valent, B. (2015). How eukaryotic filamentous pathogens evade plant recognition. Curr. Opin. Microbiol. 26, 92-101. doi: 10.1016/j.mib.2015.06.012

Palenchar, P., Kouranov, A., Lejay, L., and Coruzzi, G. (2004). Genome-wide patterns of carbon and nitrogen regulation of gene expression validate the combined carbon and nitrogen $(\mathrm{CN})$-signaling hypothesis in plants. Genome Biol. 5, 1-15. doi: 10.1186/gb-2004-5-11-r91

Penninckx, I. A. M. A., Thomma, B. P. H. J., Buchala, A., Métraux, J.-P., and Broekaert, W. F. (1998). Concomitant activation of jasmonate and ethylene response pathways is required for induction of a plant defensin gene in Arabidopsis. Plant Cell 10, 2103-2113. doi: 10.1105/tpc.10.12.2103

Ploch, S., and Thines, M. (2011). Obligate biotrophic pathogens of the genus Albugo are widespread as asymptomatic endophytes in natural populations of Brassicaceae. Mol. Ecol. 20, 3692-3699. doi: 10.1111/j.1365-294X.2011.05188.x

R Core Team (2013). R: A Language and Environment for Statistical Computing. Vienna: R Foundation for Statistical Computing. Available at: http://www.Rproject.org/

Rabello, A., Guimarães, C., Rangel, P., Da Silva, F., Seixas, D., De Souza, E., et al. (2008). Identification of drought-responsive genes in roots of upland rice (Oryza sativa L). BMC Genomics 9:485. doi: 10.1186/1471-2164-9-485

Rafiqi, M., Gan, P. H. P., Ravensdale, M., Lawrence, G. J., Ellis, J. G., Jones, D. A., et al. (2010). Internalization of flax rust avirulence proteins into flax and tobacco cells can occur in the absence of the pathogen. Plant Cell 22, 2017-2032. doi: 10.1105/tpc. 109.072983

Rappsilber, J., Mann, M., and Ishihama, Y. (2007). Protocol for micro-purification, enrichment, pre-fractionation and storage of peptides for proteomics using StageTips. Nat. Protoc. 2, 1896-1906. doi: 10.1038/nprot.2007.261

Rasmussen, S., Barah, P., Suarez-Rodriguez, M. C., Bressendorff, S., Friis, P., Costantino, P., et al. (2013). Transcriptome responses to combinations of stresses in Arabidopsis. Plant Physiol. 161, 1783-1794. doi: 10.1104/ pp.112.210773

Richards, C. L., Rosas, U., Banta, J., Bhambhra, N., and Purugganan, M. D. (2012). Genome-wide patterns of Arabidopsis gene expression in nature. PLoS Genet. 8:e1002662. doi: 10.1371/journal.pgen.1002662

Rizhsky, L., Liang, H., Shuman, J., Shulaev, V., Davletova, S., and Mittler, R. (2004). When defense pathways collide. the response of Arabidopsis to a combination of drought and heat stress. Plant Physiol. 134, 1683-1696. doi: 10.1104/pp.103.033431

Schenk, P. M., Kazan, K., Wilson, I., Anderson, J. P., Richmond, T., Somerville, S. C., et al. (2000). Coordinated plant defense responses in Arabidopsis revealed by microarray analysis. Proc. Natl. Acad. Sci. U.S.A. 97, 11655-11660. doi: 10.1073/pnas.97.21.11655

Sharon, N., and Lis, H. (2004). History of lectins: from hemagglutinins to biological recognition molecules. Glycobiology 14, 53R-62R. doi: 10.1093/glycob/cwh122

Spanu, P., and Kämper, J. (2010). Genomics of biotrophy in fungi and oomycetes - emerging patterns. Curr. Opin. Plant Biol. 13, 409-414. doi: 10.1016/j.pbi.2010.03.004
Stuttmann, J., Hubberten, H.-M., Rietz, S., Kaur, J., Muskett, P., Guerois, R., et al. (2011). Perturbation of Arabidopsis amino acid metabolism causes incompatibility with the adapted biotrophic pathogen Hyaloperonospora arabidopsidis. Plant Cell 23, 2788-2803. doi: 10.1105/tpc.111. 087684

Thomma, B. P. H. J., Eggermont, K., Penninckx, I. A. M. A., MauchMani, B., Vogelsang, R., Cammue, B. P. A., et al. (1998). Separate jasmonatedependent and salicylate-dependent defense-response pathways in Arabidopsis are essential for resistance to distinct microbial pathogens. Proc. Natl. Acad. Sci. U.S.A. 95, 15107-15111. doi: 10.1073/pnas.95.25.15107

Ton, J., and Mauch-Mani, B. (2004). $\beta$-amino-butyric acid-induced resistance against necrotrophic pathogens is based on ABA-dependent priming for callose. Plant J. 38, 119-130. doi: 10.1111/j.1365-313X.2004. 02028.x

Trentin, A. R., Pivato, M., Mehdi, S. M. M., Barnabas, L. E., Giaretta, S., FabregaPrats, M., et al. (2015). Proteome readjustments in the apoplastic space of Arabidopsis thaliana ggt1 mutant leaves exposed to UV-B radiation. Front. Plant Sci. 6:128. doi: 10.3389/fpls.2015.00128

Uknes, S., Mauch-Mani, B., Moyer, M., Potter, S., Williams, S., Dincher, S., et al. (1992). Acquired resistance in Arabidopsis. Plant Cell 4, 645-656. doi: 10.1105/tpc.4.6.645

Uknes, S., Winter, A. M., Delaney, T., Vernooij, B., Morse, A., Friedrich, L., et al. (1993). Biological induction of systemic acquired resistance in Arabidopsis. MPMI-Mol. Plant Microbe Interact. 6, 692-698. doi: 10.1094/MPMI6-692

van Treuren, R., Kuittinen, H., Kärkkäinen, K., Baena-Gonzalez, E., and Savolainen, O. (1997). Evolution of microsatellites in Arabis petraea and Arabis lyrata, outcrossing relatives of Arabidopsis thaliana. Mol. Biol. Evol. 14, 220-229. doi: 10.1093/oxfordjournals.molbev.a025758

Vorholt, J. A. (2012). Microbial life in the phyllosphere. Nat. Rev. Microbiol. 10, 828-840. doi: 10.1038/nrmicro2910

Welinder, K. G., Justesen, A. F., Kjærsgård, I. V. H., Jensen, R. B., Rasmussen, S. K., Jespersen, H. M., et al. (2002). Structural diversity and transcription of class III peroxidases from Arabidopsis thaliana. Eur. J. Biochem. 269, 6063-6081. doi: 10.1046/j.1432-1033.2002.03311.x

Wessel, D., and Flügge, U. I. (1984). A method for the quantitative recovery of protein in dilute solution in the presence of detergents and lipids. Anal. Biochem. 138, 141-143. doi: 10.1016/0003-2697(84)90782-6

Conflict of Interest Statement: The authors declare that the research was conducted in the absence of any commercial or financial relationships that could be construed as a potential conflict of interest.

Copyright (c) 2016 Ruhe, Agler, Placzek, Kramer, Finkemeier and Kemen. This is an open-access article distributed under the terms of the Creative Commons Attribution License (CC BY). The use, distribution or reproduction in other forums is permitted, provided the original author(s) or licensor are credited and that the original publication in this journal is cited, in accordance with accepted academic practice. No use, distribution or reproduction is permitted which does not comply with these terms. 